\title{
A comparison of fixed and dynamic pricing policies in revenue management
}

\author{
Alper Șen* \\ Department of Industrial Engineering, Bilkent University, Bilkent, Ankara 06800, Turkey
}

\section{A R T I C L E I N F O}

\section{Article history:}

Received 22 September 2011

Accepted 10 August 2012

Processed by B. Lev

Available online 28 August 2012

Keywords:

Dynamic pricing

Revenue management

Yield management

Heuristics

\begin{abstract}
A B S T R A C T
We consider the problem of selling a fixed capacity or inventory of items over a finite selling period. Earlier research has shown that using a properly set fixed price during the selling period is asymptotically optimal as the demand potential and capacity grow large and that dynamic pricing has only a secondary effect on revenues. However, additional revenue improvements through dynamic pricing can be important in practice and need to be further explored. We suggest two simple dynamic heuristics that continuously update prices based on remaining inventory and time in the selling period. The first heuristic is based on approximating the optimal expected revenue function and the second heuristic is based on the solution of the deterministic version of the problem. We show through a numerical study that the revenue impact of using these dynamic pricing heuristics rather than fixed pricing may be substantial. In particular, the first heuristic has a consistent and remarkable performance leading to at most $0.2 \%$ gap compared to optimal dynamic pricing. We also show that the benefits of these dynamic pricing heuristics persist under a periodic setting. This is especially true for the first heuristic for which the performance is monotone in the frequency of price changes. We conclude that dynamic pricing should be considered as a more favorable option in practice.
\end{abstract}

(c) 2012 Elsevier Ltd. All rights reserved.

\section{Introduction}

Pricing is one of the most important decisions that impact a firm's profitability. The effect of pricing is more profound for companies in transportation services sector where it is difficult to change capacities in the short term and variable costs are small. Recognizing this, airlines, rental car companies and other firms in transportation and service industries have begun to implement techniques to improve their pricing and allocation decisions since mid 1980s. Following the success of these practices, now broadly called revenue management, pricing decisions are becoming more tactical and dynamic pricing is increasingly being adopted in retail and other industries.

In a seminal work, Gallego and van Ryzin [1] (GvR hereafter) study the problem of dynamically pricing a fixed stock of items over a finite horizon under uncertain demand. An important result in GvR is that keeping the price constant (at a level determined by the deterministic solution of the problem) throughout the horizon has a bounded worst-case performance and is asymptotically optimal as the expected sales goes to infinity. GvR also show numerically that when the demand function is exponential, fixed-price policies have good performance even when the expected sales is small. The authors

\footnotetext{
* Tel.: +90 312290 1539; fax: +90312266 4054 .

E-mail address: alpersen@bilkent.edu.tr
}

conclude that “... offering multiple prices can at best capture only second-order increases in revenue due to the statistical variability in demand". Since 1994, a large and important body of literature in operations research has evolved to offer solutions and study different variants of the problem studied in GvR. (Recent examples include research that study the impact of product substitution [2], consumer inertia [3] and competition and price uncertainty [4] on dynamic pricing. See [5-7] for extended reviews of earlier literature.) Although GvR caution that these second-order increases in revenue may be significant in practice, revenue management literature has remained relatively silent on quantifying the benefits of dynamic pricing over fixed-price policies. This is primarily due to practical convenience: computing optimal dynamic prices is difficult (if not impossible) and changing prices frequently may be undesirable or costly.

Our primary aim in this paper is to reemphasize the power of dynamic pricing under resupply restrictions. We suggest two computationally simple dynamic pricing heuristics and show that the performance of these heuristics can be significantly better than that of fixed-price policies. In particular, we first propose the revenue approximation heuristic which is based on approximating the expected revenue of the optimal policy in order to calculate the price to be applied for a given remaining inventory and remaining time in the selling season. The approximation is a combination of a lower bound based on the homogeneity of the optimal expected revenue and an upper bound based on the deterministic version of the problem. The second heuristic we 
suggest is the dynamic run-out rate heuristic which adaptively uses the solution of the deterministic version of the problem. We carry out an extensive numerical study which shows that the revenue gap between fixed-price and optimal dynamic pricing policies may be substantial and this gap worsens when the season length (or demand potential) increases. We show that the two heuristics that we propose close a significant portion of this gap and lead to near-optimal expected revenues. We also show that most of the benefits of dynamic pricing heuristics are sustained by changing the prices periodically rather than continuously. For the first heuristic, the performance is monotone in the number of periods used. Our analysis and results are confined to the benefits of dynamic pricing under "normal" statistical fluctuations in demand. The benefits of dynamic pricing will be more pronounced when the demand is non-homogeneous or when the demand function or distribution is not known in advance.

Among the relevant works in the literature, Gallego and van Ryzin [8] extend their model to the multiple products case and demonstrate that two heuristics that are similarly based on the solution of the deterministic version of the problem are asymptotically optimal. Cooper [9] proves asymptotical convergence results that are stronger than those in GvR and [8]. Cooper [9] also presents an example where updating prices (more precisely, the allocations in Cooper's model) by resolving the deterministic problem throughout the horizon, a widely applied approach in practice, may perform worser than applying the static policy. Secomandi [10] establishes the conditions under which resolving does not deteriorate the performance of heuristic pricing policies. Maglaras and Meissner [11] show that resolving heuristics are also asymptotically optimal as starting inventory and expected sales both go to infinity and Cooper's example should not persist in problems with large demand potential. There is limited research on developing dynamic pricing heuristics and those that are suggested are usually based on deterministic formulations. The main contribution in this paper is to propose two new heuristics that are simple and computationally feasible. While dynamic run-out rate heuristic also uses the deterministic solution in feedback form, revenue approximation heuristic is based on approximating the revenue-to-go function using a homogeneity assumption.

The literature also does not provide enough guidance on nonasymptotic or average performance of heuristic policies and the factors that moderate their performance. In GvR, the authors use the exponential price sensitivity of demand and conduct a small numerical experiment to study the performance of the fixed-price policy against the optimal dynamic policy. It is shown that the revenue gap between the fixed-price and dynamic pricing policies is smaller than the theoretical bounds and gets smaller as starting inventory increases. However, Zhao and Zheng [12] show that the revenue gap is more significant when the constant demand elasticity function is used rather than the exponential demand function. Zhao and Zheng [12] also show that the revenue gap is rather insensitive to the elasticity of demand and there are diminishing marginal returns of dynamic pricing policies to the number of prices used. Maglaras and Meissner [11] conduct a numerical study on the multiproduct pricing problem with a linear demand function. Their results show that the fixed-price policy's regret over the optimal dynamic policy can be substantial and resolving the deterministic problem periodically during the horizon can offer significant benefits. In Section 3, we provide the results of an extensive numerical experiment to study the performance of heuristic pricing policies. The results show that the regret of fixed-price policies can be important in practice and dynamic pricing heuristics can be used to generate near-optimal results.

The remainder of this paper is organized as follows. In Section 2, we propose the revenue approximation and dynamic run-out rate
Table 1

The demand functions that are used in the analysis.

\begin{tabular}{llllll}
\hline & $\lambda(p)$ & $p(\lambda)$ & $r(\lambda)$ & $\lambda^{*}$ & $p^{*}$ \\
\hline Exponential & $a e^{-p}$ & $\ln \left(\frac{a}{\lambda}\right)$ & $\lambda \ln \left(\frac{a}{\lambda}\right)$ & $\frac{a}{e}$ & 1 \\
Linear & $a-b p$ & $\frac{a-\lambda}{b}$ & $\frac{(a-\lambda) \lambda}{b}$ & $\frac{a}{2}$ & $\frac{a}{2 b}$ \\
Logit & $\frac{a e^{-b p}}{1+e^{-b p}}$ & $\frac{1}{b} \ln \left(\frac{a}{\lambda}-1\right)$ & $\frac{\lambda}{b} \ln \left(\frac{a}{\lambda}-1\right)$ & $\frac{a e^{-W(1 / e)-1}}{1+e^{-W(1 / e)-1}}$ & $\frac{W(1 / e)+1}{b}$
\end{tabular}

heuristics. In Section 3, we report the results of a detailed numerical study that quantifies the regrets of fixed-price and dynamic pricing heuristics over the optimal dynamic pricing policy. This section also analyzes the effect of periodic price changes on the performance of dynamic pricing heuristics. We conclude in Section 4.

\section{Dynamic pricing heuristics}

We first state our problem following the notation in GvR and provide some preliminary results. A given stock of $n$ items is to be sold over a finite season of length $t$. The demand rate depends only on the current price $p$ through a function $\lambda(p)$, whose inverse is $p(\lambda)$. The revenue rate, denoted by $r(\lambda)=\lambda p(\lambda)$, is assumed to satisfy $\lim _{\lambda \rightarrow 0} r(\lambda)=0$, and is continuous, bounded, concave and has a least maximizer denoted by $\lambda^{*}=\min \left\{\lambda: r(\lambda)=\max _{\lambda \geq 0} r(\lambda)\right\}$ (the corresponding price is $p^{*}=p\left(\lambda^{*}\right)$ ). There exists a null price denoted by $p_{\infty}$ for which $\lim _{p \rightarrow p_{\infty}} \lambda(p)=0$. The price is selected from a set of allowable prices $\mathcal{P}=\mathbb{R}_{+} \cup p_{\infty}$. The corresponding set of allowable rates is denoted by $\Lambda=\{\lambda(p): p \in \mathcal{P}\}$.

For the numerical examples and experiments in this paper, we use three different functions to model the price-demand relationship: exponential, linear and logit demand functions. These are some of the most commonly used demand functions in theory and practice $[7,13]$ and are given in Table $1 .{ }^{1}$

The demand is stochastic and modeled as a Poisson Process. The firm controls the intensity at every instant by using a price in $\mathcal{P}$. The problem is to determine the pricing policy that maximizes the total expected revenue over the season denoted by $J^{*}(n, t)$.

For a given remaining time $s$ and inventory $x$ in the season, GvR show that the optimal expected revenue-to-go (and the corresponding optimal price at that instant) can be found by solving the following system of differential

$\frac{\partial J^{*}(x, s)}{\partial s}=\sup _{\lambda}\left\{r(\lambda)-\lambda\left(J^{*}(x, s)-J^{*}(x-1, s)\right)\right\}, \quad$ for all $x=1,2, \ldots, n$,

with boundary conditions $J^{*}(x, 0)=0$ for all $x=1,2, \ldots, n$ and $J^{*}(0, s)=0$ for all $s \leq t$. GvR also prove the existence of a unique solution to (1) along with monotonicity of the optimal expected revenue (and corresponding demand rates and prices) with respect to remaining inventory and remaining time in the season.

GvR state that obtaining a solution to (1) is quite difficult - if not impossible - for arbitrary demand functions. In addition, implementing a pricing policy that would change the price continuously over time may be difficult in practice. Therefore, they suggest the use of a heuristic pricing policy in which the price is constant for the entire season. The fixed-price (FP) heuristic that they develop uses the solution of the deterministic version of the problem and sets the price at $\bar{p}=p(\bar{\lambda})=p\left(\min \left\{\lambda^{0}, \lambda^{*}\right\}\right)$, where $\lambda^{0}=n / t$ is the runout rate and $\lambda^{*}$ is the revenue maximizing rate. One can improve

${ }^{1} W($.$) denotes the principal branch of the Lambert W$ function, which is the inverse of the function $f(w)=w e^{w}$. The numeric value of $W(1 / e)$ is approximately 0.27846 . 
upon this by using the optimal fixed-price (OFP) heuristic and setting the price to $p_{O F P}=\arg \max _{p} p \mathbb{E}\left[\min \left\{n, N_{\lambda(p)}(t)\right\}\right]$ where $N_{\lambda(p)}(t)$ is a Poisson random variable with rate $\lambda(p) t$. GvR shows that both heuristic are asymptotically optimal as $n$ and $\lambda^{*} t$ (or demand potential) both go to infinity. In the remainder of the section, we suggest two computationally simple heuristics that can be used to dynamically adjust prices.

\subsection{Revenue approximation heuristic}

The main idea behind our first heuristic approach is to approximate the optimal expected revenue function $J^{*}$ with a proper function, say $\tilde{J}$, and use this approximation in (1) to find

$\lambda_{R A}(x, s)=\arg \sup \{r(\lambda)-\lambda(\tilde{J}(x, s)-\tilde{J}(x-1, s))\}$.

This is similar to the approximate dynamic programming approach used in [14] and [15] to calculate bid prices for network revenue management by approximating the value function in Bellman equation. Zhang and Cooper [16] use a similar approach to determine prices in a revenue management problem with substitutable flights. Our approach differs from theirs as we use a new way to approximate the value function and consider a continuous time dynamic program (thus use approximation in the Hamilton-Jacobi optimality condition). We first develop a lower bound and an upper bound for the value function and then use a combination of these bounds to approximate the value function.

\subsubsection{Lower bound}

The lower bound we develop is based on the following intuitively appealing argument: The optimal expected revenue that can be obtained by selling $x$ units of remaining inventory over a remaining season of length $s$ is approximately equal to $x$ times the optimal expected revenue that can be obtained by selling one unit of inventory over a season of length $x / s$, i.e.,

$\tilde{J}_{H}(x, s)=x J^{*}(1, s / x)$.

This approximation would be exact only if the optimal expected revenue function was positively homogeneous, i.e., $J^{*}(x, s)=x J^{*}(1, s / x)$. As we show next, this is not the case and the expected revenue obtained through this approximation is a lower bound for the optimal expected revenue.

\section{Theorem 1.}

$\tilde{J}_{H}(x, s)=x J^{*}(1, s / x) \leq J^{*}(x, s), \quad \forall x>0$.

Proof. Consider the pricing policy for $x$ units of inventory to be sold over a remaining season of length $s$. The remaining season is split into $x$ periods, each having length $s / x$. In each of these periods, one additional inventory is put on sale along with any leftover inventory from the previous period. In each period, the intensity at time $w$ is set to $\lambda^{*}(1,(s / x)-w)$. Since $J^{*}(1, s / x)$ is the expected revenue of this policy in one of these periods without considering the leftover inventory, there is a positive probability (which is equal to or larger than $e^{m_{x}(s)}$ where $m_{x}(s)=\int_{0}^{s / x}$ $\left.\lambda^{*}(1,(s / x)-w) d w\right)$ that there will be leftover inventory at the end of a given period, and the prices are non-zero, the expected revenue resulting from this pricing policy is at least $x J^{*}(1, s / x)$.

Fig. 1 shows the percentage gap between the lower bound and the optimal solution given by

$100 \times \frac{J^{*}(x, s)-\tilde{J}_{H}(x, s)}{J^{*}(x, s)}$

for the exponential, linear, and logit demand functions for $x=2,5,10$. We take $a=e$ for the exponential, $(a, b)=(2,1)$ for the linear and $(a, b)=\left(1+e^{-W(1 / e)-1} / e^{-W(1 / e)-1}, W(1 / e)+1\right)$ for the logit demand functions leading to $p^{*}=\lambda^{*}=1$ for all demand functions.

The gaps tend to be small for small $s$, but increase rapidly to their peak at moderate $x$ values and then stabilize. We see a similar pattern for different parameter values as well.

The lower bound requires the calculation of $J^{*}(1, s)$ using a single differential equation

$\frac{\partial J^{*}(1, s)}{\partial s}=\sup _{\lambda}\left\{r(\lambda)-\lambda J^{*}(1, s)\right\}$.

Remember that obtaining the optimal policy requires solving the system of differential equations given in (1). Therefore, obtaining the lower bound is much simpler compared to the optimal policy. For $x=1$, the lower bound coincides with the optimal expected revenue, i.e., $\tilde{J}_{H}(1, s)=J^{*}(1, s)$.

\subsubsection{Upper bound}

The upper bound we use is the solution of the problem in which the demand rates are deterministic. In this case, as is shown in [1], we have:

$\tilde{J}_{D}(\chi, s)=r(\bar{\lambda}(\chi, s)) s=r\left(\min \left\{\lambda_{0}(\chi, s), \lambda^{*}\right\}\right) s$,

where $\lambda_{0}(x, s)=x / s$ is the run-out rate. As shown below, $\tilde{J}_{D}(x, s)$ constitutes an upper bound for the optimal revenue $J^{*}(x, s)$.

Theorem 2 (Gallego and van Ryzin [1, Theorem 2]).

$J^{*}(x, s) \leq \tilde{J}_{D}(x, s), \quad \forall x>0$.

\subsubsection{Approximation}

Since we establish $\tilde{J}_{H}(x, s) \leq J^{*}(x, s) \leq \tilde{J}_{D}(x, s)$ in Theorems 1 and 2, we can obtain better approximations for the optimal revenue through a combination of $\tilde{J}_{H}(x, s)$ and $\tilde{J}_{D}(x, s)$,

$\tilde{J}(x, s)=\theta(x, s) \tilde{J}_{H}(x, s)+(1-\theta(x, s)) \tilde{J}_{D}(x, s)$.

In principal, $\theta(x, s)$ can be fine-tuned for a given demand function, starting inventory and length of the horizon. For example, Fig. 2 shows the optimal revenue as well as the upper and lower bounds for the linear demand function with $a=2$ and $b=1$. As one can observe, the lower bound is tighter than the upper bound for small values of starting inventory, but the upper bound better approximates the optimal revenue for larger values of starting inventory. In Section 3, we use the weights $\theta(x, s)=1 / \sqrt{x}$ in a detailed numerical study. This leads to a heuristic performance within or around $0.2 \%$ of the optimal revenue for all problems we consider.

We now explain how one can compute the intensity and corresponding prices for the revenue approximation heuristic for the three demand functions used in this paper.

Exponential demand function: For the exponential demand function, using (2), we get

$$
\lambda_{R A}(x, s)=\frac{a}{e^{1+\tilde{J}(x, s)-\tilde{J}(x-1, s)}} .
$$

For the exponential demand function, $J^{*}(1, s)=\ln \left(1+\lambda^{*} s\right.$ ) (see GvR). Therefore, we have $\tilde{J}_{H}(x, s)=x \ln \left(1+\lambda^{*} s / x\right)$. In addition, $\tilde{J}_{D}(x, s)=\min \left\{x, \lambda^{*} s\right\} \ln \left(\right.$ as $\left./ \min \left\{x, \lambda^{*} s\right\}\right)$. Using these in (4),

$$
\lambda_{R A}(x, s)= \begin{cases}\frac{a}{e\left(1+\lambda^{*} s\right)^{\theta(1, s)}\left(\frac{a s}{\min \left\{1, \lambda^{*} s\right\}}\right)^{\min \left\{1, \lambda^{*} s\right\}(1-\theta(1, s))}} & \text { if } x=1, \\ \frac{a\left(1+\frac{\lambda^{*} s}{x-1}\right)^{(x-1) \theta(x, s)}\left(\frac{a s}{\min \left\{x-1, \lambda^{*} s\right\}}\right)^{\min \left\{x-1, \lambda^{*} s\right\}(1-\theta(x-1, s))}}{e\left(1+\frac{\lambda^{*} s}{x}\right)^{x \theta(x, s)}\left(\frac{a s}{\min \left\{x, \lambda^{*} s\right\}}\right)^{\min \left\{x, \lambda^{*} s\right\}(1-\theta(x-1, s))}} & \text { if } x \geq 2,\end{cases}
$$

where $\lambda^{*}=a / e$. The corresponding price is $p_{R A}(x, s)=\ln \left(a / \lambda_{R A}(x, s)\right)$. Note that the optimal price and intensity can be calculated in closed form. The optimal price is an increasing (decreasing) 

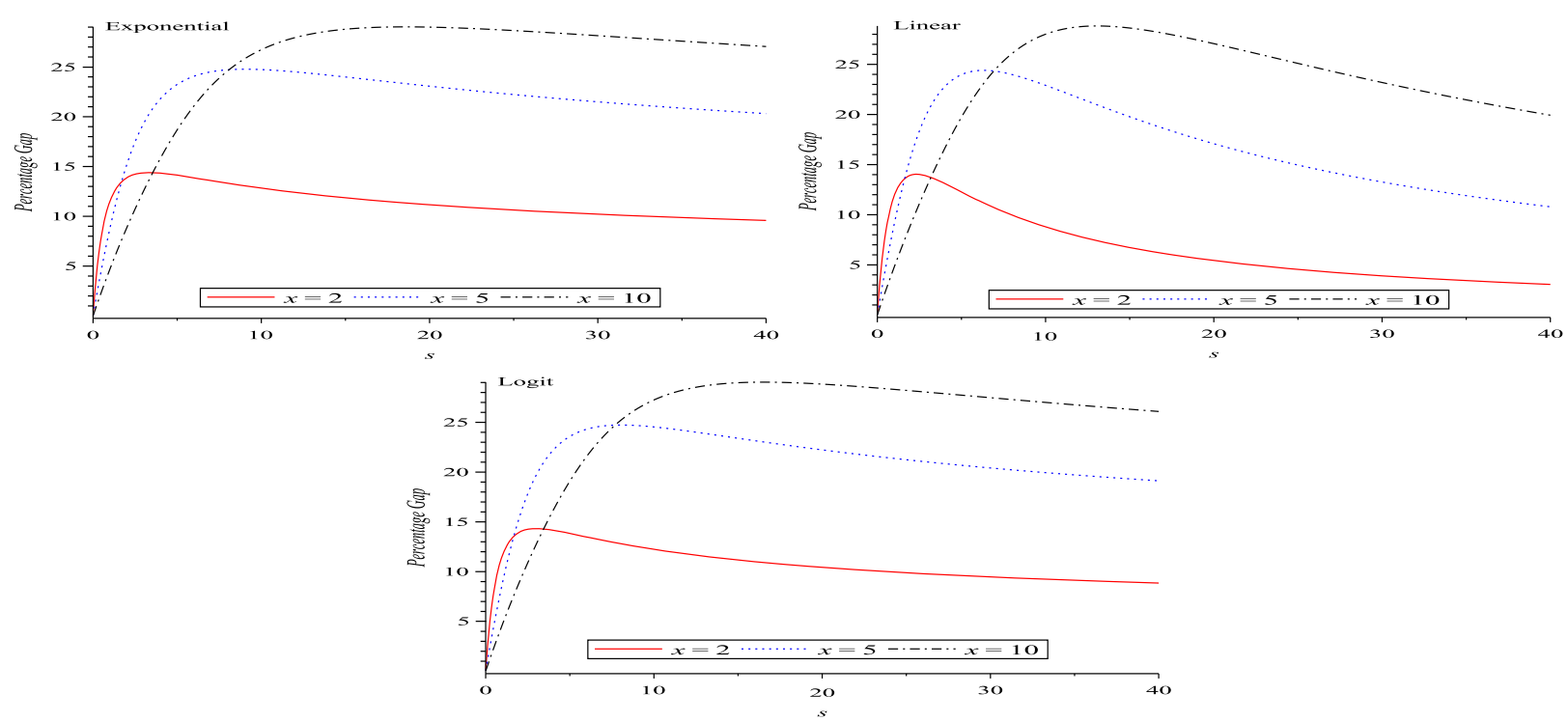

Fig. 1. Percentage gap of the lower bound for $p^{*}=\lambda^{*}=1$.
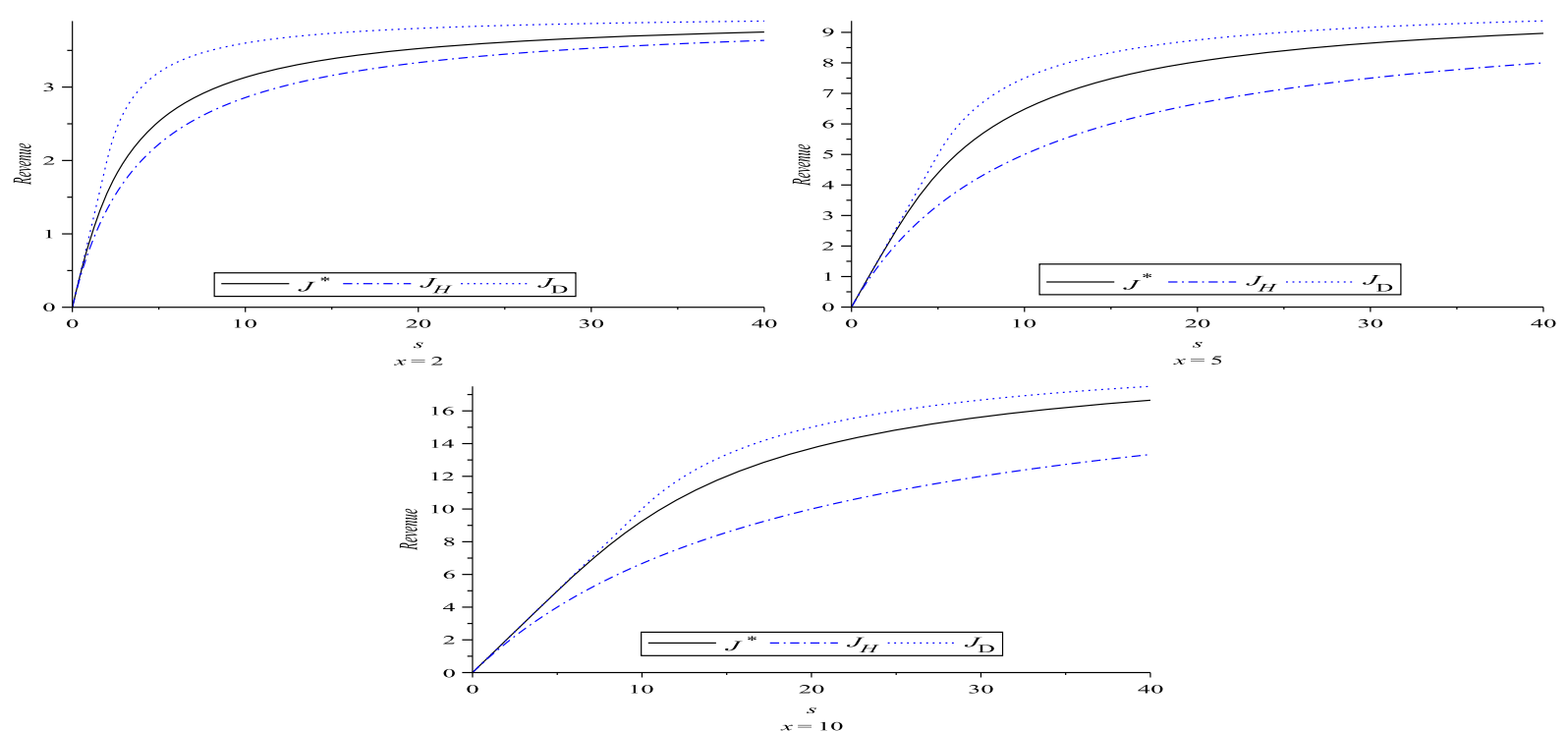

Fig. 2. Upper and lower bounds for the optimal revenue for the linear demand function with $a=2$ and $b=1$.

function of the remaining time (inventory) in the season. Correspondingly, optimal intensity is a decreasing (increasing) function of the remaining time (inventory) in the season.

Linear demand function: For the linear demand function, using (2), we get

$$
\lambda_{R A}(x, s)=\frac{a}{2}-\frac{b}{2}(\tilde{J}(x, s)-\tilde{J}(x-1, s))
$$

In order to find $\tilde{J}_{H}(x, s)$, one needs to first calculate $J^{*}(1, s)$. By solving (3), we get,

$J^{*}(1, s)=\frac{a^{2} s}{b(a s+4)}$.

Therefore, we have $\tilde{J}(x, s)=a^{2} s x / b(a s+4 x)$. In addition, $\tilde{J}_{D}(x, s)=\min \left\{x, \lambda^{*} s\right\}\left(a s-\min \left\{x, \lambda^{*} s\right\}\right) / b s$ where $\lambda^{*}=a / 2$. Then, we get

$$
\lambda_{R A}(x, s)=\left\{\begin{array}{cc}
\frac{a}{2}-\frac{a^{2} s \theta(1, s)}{2(a s+4)}-\frac{\min \left\{1, \frac{a s}{2}\right\}\left(a s-\min \left\{1, \frac{a s}{2}\right\}\right)(1-\theta(1, s))}{2 s} & \text { if } x=1, \\
\frac{a}{2}-\frac{a^{2} s \theta(x, s) x}{2(a s+4 x)}+\frac{a^{2} s \theta(x-1, s)(x-1)}{2(a s+4(x-1))} & \\
-\frac{\min \left\{x, \frac{a s}{2}\right\}\left(a s-\min \left\{x, \frac{a s}{2}\right\}\right)(1-\theta(x, s))}{2 s} & \text { if } x \geq 1 . \\
+\frac{\min \left\{x-1, \frac{a s}{2}\right\}\left(a s-\min \left\{x-1, \frac{a s}{2}\right\}\right)(1-\theta(x-1, s))}{2 s} &
\end{array}\right.
$$

The corresponding price is $p_{R A}(x, s)=a-\lambda_{R A}(x, s) / b$. Again, the optimal price and intensity can be written in closed form and maintain monotonicity properties. 
Logit demand function: For the logit demand function, using (2), we get

$\lambda_{R A}(x, S)=\frac{a}{1+e^{\mathrm{W}\left(e^{-b \tilde{J}(x, s)-\tilde{J}(x-1, S))-1}\right)+b(\tilde{J}(x, s)-\tilde{J}(x-1, s))+1} .}$

The corresponding price is $p_{R A}(x, s)=\frac{1}{b} \ln \left(a / \lambda_{R A}(x, s)-1\right)$. The solution to the deterministic problem leads to $\tilde{J}_{D}(x, s)=(\mathrm{min}$ $\left.\left\{x, \lambda^{*} s\right\} / b\right) \ln \left(\left(a s / \min \left\{x, \lambda^{*} s\right\}\right)-1\right) \quad$ where $\quad \lambda^{*}=a e^{-W(1 / e)-1} / 1+$ $e^{-W(1 / e)-1}$. Unfortunately, however, there is no closed-form representation of $\tilde{J}_{H}(x, s)$ since there is no closed-form solution for $J^{*}(1, s)$ in $(3) . J^{*}(1, s)$ can only be represented as a solution $(z)$ to the following equation.

$\int_{0}^{z} \frac{1+\mathrm{W}\left(e^{-b y-1}\right)}{\mathrm{W}\left(e^{-b y-1}\right)\left[\ln \left(\frac{1}{\mathrm{~W}\left(\mathrm{e}^{-b y-1}\right)}\right)-b y\right]} d y=\frac{a x}{b}$.

Therefore, all calculations need to be carried out numerically by obtaining the solution $J^{*}(1, s)$ from $(6)$ to get $\tilde{J}_{H}(x, s)=x J^{*}(1, s / x)$. However, the computation burden of the heuristic is much less compared to obtaining the solutions for $J^{*}(x, s)$ for all $x=1, \ldots, n$.

In general, calculating the prices (or intensities) that will be used for $R A$ heuristic is as difficult as solving the single differential in (3) and if (3) has a closed-form solution, the prices can also be represented in closed form.

One can extend the idea used in computing the lower bound to a class of dynamic pricing heuristics by approximating $J^{*}(x, s)$ with $\tilde{J}_{H}(x, s)=(x / k) J^{*}(k, s k / x)$ with $k \geq 1$. More generally, one can use a linear combination of $d$ of these approximations such that $\tilde{J}_{H}(x, s)=\sum_{k=1}^{d} \alpha_{k}(x / k) J^{*}(k, s k / x)$. We performed a preliminary numerical investigation of the performance of these heuristics with $d>1$, but since this leads to additional computational burden and does not necessarily provide a tighter bound in our numerical study, we only focus on $d=1$ and $\alpha_{1}=1$ in this paper.

\subsection{Dynamic run-out rate heuristic}

The dynamic run-out rate heuristic is a dynamic version of $F P$ heuristic suggested in GvR. For a given remaining time $s$ in the horizon and remaining inventory $x$, the price is set at

$p_{R R}(x, s)=\bar{p}(x, s)=\max \left\{p^{*}, p^{0}(x, s)\right\}$,

where $p^{*}$ is the revenue maximizing price and $p^{0}(x, s)=p\left(\lambda^{0}(x, s)\right)$ with $\lambda^{0}(x, s)=x / s$ being the run-out rate. Alternatively, this heuristic sets the intensity at

$\lambda_{R R}(x, s)=\bar{\lambda}(x, s)=\min \left\{\lambda^{*}, \lambda^{0}(x, s)\right\}$.

Note that $p_{R R}(x, s)$ is the solution of the deterministic version of the problem solved when the remaining time in the season is $s$ and remaining inventory is $x$. Thus, this heuristic is equivalent to continuously "resolving" the deterministic problem (fluid policy).

It is worthwhile here to note what distinguishes dynamic run-out rate heuristic $(R R)$ from fixed-price $(F P)$ heuristic. $F P$ heuristic solves the deterministic problem once only at the beginning of the selling period when there are $n$ units of inventory and $t$ units of time remaining. This leads to the price $p_{F P}(n, t)=\max \left\{p^{*}, p\left(\lambda^{0}(n, t)\right)\right\}$, where $\lambda^{0}(n, t)=n / t$ is the run-out rate. FP does not change this price during the selling period. $R R$ heuristic, on the other hand, resolves the deterministic problem at every instant by recalculating run-out rate $\lambda^{0}(x, s)=x / s$ for the given remaining time $s$ and inventory $x$, and sets the price to $p_{R R}(x, s)=\max \left\{p^{*}, p\left(\lambda^{0}(x, s)\right)\right\}$ at that instant.

Example price paths: We demonstrate the price paths created by the optimal and heuristic policies in an example in Fig. 3. There are $n=5$ units of inventory to sell over a horizon of length $t=10$. The average demand rate depends on the price through the function $\lambda(p)=2-p$ (linear price response function with $a=2$ and $b=1)$. For this function, we have, $p^{*}=\lambda^{*}=1$. FP heuristic sets the price to $p_{F P}=\bar{p}=p\left(\min \left\{\lambda^{*}, n / t\right\}\right)=p(\min \{1,0.5\})=2-0.5=1.5$. One can determine the price of OFP heuristic by maximizing $p \mathbb{E}[\mathrm{min}$ $\left.\left\{n, N_{\lambda(p)}(t)\right\}\right]=p \mathbb{E}\left[\min \left\{5, N_{2-p}(10)\right\}\right]$. A numerical procedure can be used to find $p_{\text {OFP }}=1.419305$. Dynamic pricing policies adjust the price as a function of remaining time $s$ and remaining inventory $x$. $R R$ heuristic sets the price to $p_{R R}(x, s)=p\left(\min \left\{\lambda^{*}, x / s\right\}\right)=2$ $\min \{1, x / s\}$. As explained in Section 2.1, $R A$ heuristic computes a lower and an upper bound for the revenue-to-go and uses a combination of these to compute the price. In this example (as well as in most of other numerical experiments), we use $\theta(x, s)=1 / \sqrt{x}$ as the weight of the lower bound. Using this, $\lambda_{R A}(x, s)$ given in (5) and the fact that $p_{R A}(x, s)=2-\lambda_{R A}(x, s)$, we find

$p_{R A}(x, s)= \begin{cases}1+\frac{2 s \sqrt{x}}{2 s+4 x}-\frac{2 s \sqrt{x-1}}{2 s+4(x-1)}+\frac{\min \{x, s\}(2 s-\min \{x, s\})(\sqrt{x}-1)}{2 s \sqrt{x}} & \\ -\frac{\min \{x-1, s\}(2 s-\min \{x-1, s\})(\sqrt{x-1}-1)}{2 s \sqrt{x-1}} & \text { if } x>1, \\ 1+\frac{2 s}{2 s+4} & \text { if } x=1 .\end{cases}$

Finally, the optimal dynamic price $p^{*}(x, s)$ can be computed only numerically by solving the system of differential equations given in (1).

Sample price paths for optimal dynamic pricing (denoted by $O P T), R A$ heuristic and $R R$ heuristic are plotted in Fig. 3, as well as the fixed prices set by FP and OFP heuristics. The horizontal axis represents the remaining time in the season. The jumps in dynamic policies correspond to sales (for demonstration, the example assumes that the sales are realized at the same times for each policy, although, in reality the realizations depend on the prices charged and hence could be different for each policy). As is the case for the optimal dynamic policy, both dynamic pricing heuristics

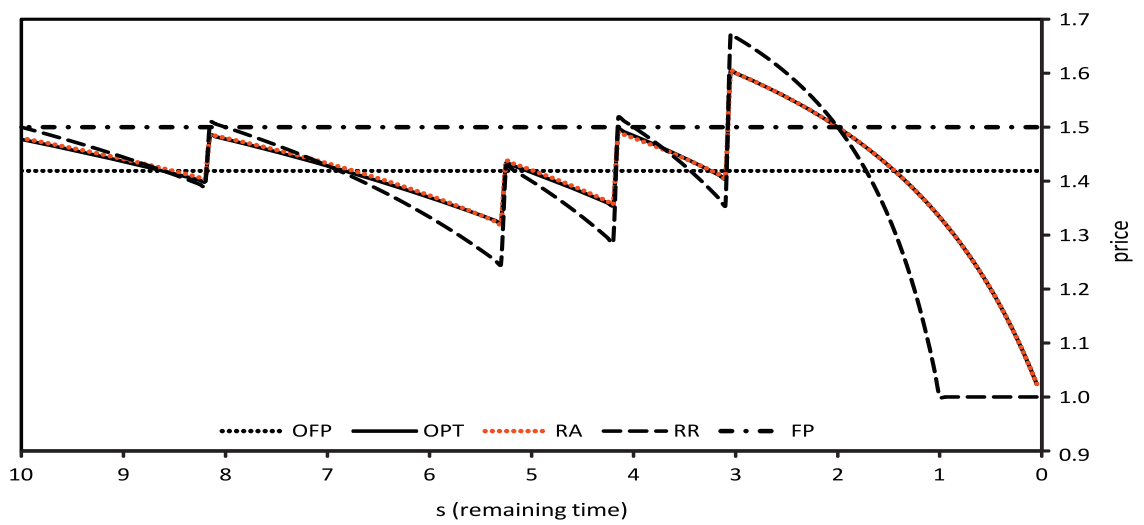

Fig. 3. Price paths for optimal and heuristic policies, linear demand, $a=2, b=1, t=10, n=5$. 


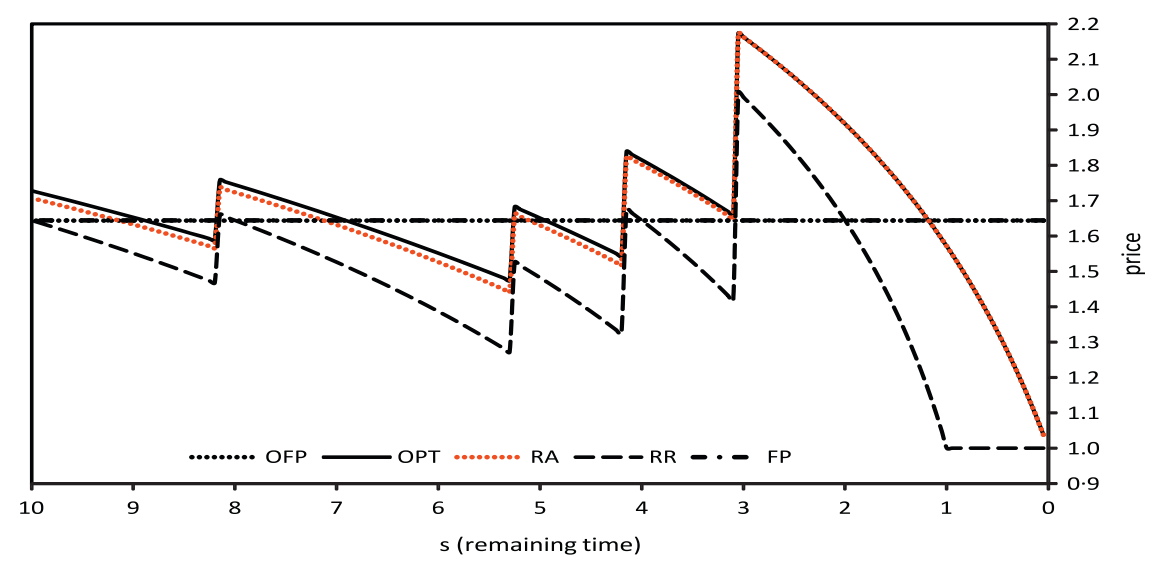

Fig. 4. Price paths for optimal and heuristic policies, logit demand, $b=W(1 / e), a=1+e^{-W(1 / e)-1} / e^{-W(1 / e)-1}, t=10, n=5$.

reduce the price over time between consecutive sales and introduce an upward jump at each sale (the only exception to this behavior is when the remaining time in the selling period is less than 1 and remaining inventory is 1 , leading to a constant price $p_{R R}(1, s)=$ $p(\min \{1,1 / s\})=1$ for $R R$ heuristic). The price set by $R R$ heuristic can be somewhat different from the optimal price. On the other hand, $R A$ heuristic's price is always very close to the optimal dynamic price. In this particular case, the difference $p^{*}(x, s)-p_{R A}(x, s)$ remains in the interval $[-0.005017,0.005708]$. The optimal expected revenue for this example is $J^{*}(5,10)=6.4857$. Using $R A, R R$, OFP, FP heuristics instead generate expected revenues $J_{R A}(5,10)=6.4844, J_{R R}(5,10)=$ $6.4268, J_{O F P}(5,10)=6.2795, J_{F P}(5,10)=6.1840$.

Fig. 4 shows similar price paths for an example with logit price response function with parameters $b=W(1 / e)$ and $a=1+$ $e^{-W(1 / e)-1} / e^{-W(1 / e)-1}$ leading to $p^{*}=\lambda^{*}=1$. Again, we have five units of inventory to sell over a selling period of 10 time units. In this case, FP and OFP heuristics' prices are very close to each other; $p_{F P}=1.6441$ and $p_{O F P}=1.6439$.

The price paths for the optimal dynamic policy and dynamic heuristics have shapes similar to those in Fig. 3. However, in this case the range of prices are larger. $R A$ heuristic still follows the optimal policy closely although not as closely as the case for linear price response function. Again, $R R$ heuristic may set a price quite different from what is optimal. The optimal expected revenue for this example is $J^{*}(5,10)=7.0737$. Using $R A, R R, O F P$, $F P$ heuristics instead generate expected revenues $J_{R A}(5,10)=$ $7.0711, J_{R R}(5,10)=6.9535, J_{O F P}(5,10)=6.7782, J_{F P}(5,10)=6.7782$.

\section{Numerical study}

In this section, we analyze the performance of dynamic pricing heuristics (namely, revenue approximation $(R A)$ and dynamic run-out rate $(R R)$ heuristics) and compare their performance against constant price heuristics (namely, fixed-price $(F P)$ and optimal fixed-price $(O F P)$ heuristics) through a detailed numerical study. We also attempt to complement the numerical analysis in GvR for FP and OFP by considering different demand functions and larger demand potentials. For this purpose, we use exponential, linear and logit demand functions.

In order to calculate the expected revenue of a given dynamic pricing heuristic $P$, we first numerically solve the system:

$\frac{\partial J_{P}(x, s)}{\partial s}=r\left(\lambda_{P}(x, s)\right)-\lambda_{P}(x, s)\left[J_{P}(x, s)-J_{P}(x-1, s)\right]$, for all $x=1, \ldots, n$,

with initial conditions $J_{P}(0, s)=0, \forall s$ and $J_{P}(x, 0)=0$, for all $x=1, \ldots, n$, where $\lambda_{P}(x, s)$ is the demand rate set by the heuristic policy. The expected revenue of using the heuristic policy $P$ then can be found by evaluating $J_{P}(x, s)$ at $x=n$ and $s=t$.

In order to calculate the optimal revenue $J^{*}(n, t)$, we solve the system of differential equations (1) numerically. We carried out these calculations in an advanced numerical mathematics software package. For larger problems (especially for larger values of starting inventory level) or more complex price-response functions, obtaining the optimal policy may be intractable or the computation times may be prohibitive in a practical setting.

\subsection{Performance of fixed and dynamic pricing policies}

In Table 2, we report the optimal revenue and performance of heuristic policies for the exponential demand function when $n=1, \ldots, 20$ and $\lambda^{*} t$ takes on values 10 or 40 . The first four columns of Table 2 report the optimal expected revenue $\left(J^{*}\right)$ and the performance of fixed price policies FP and OFP for $\lambda^{*} t=10$. These are exactly same as what is reported in Table 1 of GvR. We extend the numerical study in GvR for a larger demand potential $\left(\lambda^{*} t=40\right)$ in columns $8-10$. In addition, we report the performance of heuristic dynamic pricing policies. $J_{R R}$ denotes expected revenue of the dynamic run-out heuristic. $J_{R A}^{D}$ denotes the expected revenue of the revenue approximation heuristic when only the deterministic upper bound is used to approximate the value function (i.e., $\theta(x, s)=0), J_{R A}^{H}$ denotes the expected revenue of the revenue approximation heuristic when only the lower bound is used (i.e., $\theta(x, s)=1$ ) and $J_{R A}^{H}$ denotes the expected revenue approximation heuristic when weights are set to $\theta(x, s)=1 / \sqrt{x}$ (We investigated the use of other weights such as $\theta(x, s)=0.5$ or other functional forms, but these did not lead to better performance).

When $\lambda^{*} t=10$, the regrets of FP and OFP heuristics are relatively small. FP heuristic performs worst at $87.06 \%$ for $n=1$, but for larger values of $n$, the performance is good and approaches $100 \%$ when $n=20$. OFP heuristic's worst performance is $94.51 \%$. Comparing columns 3 and 4 with columns 10 and 11 shows that both FP and OFP heuristics perform worse for all, but two values of $n$ when $\lambda^{*} t=40$ case. Average reduction in performance is $3.15 \%$ and $2.85 \%$ for FP and OFP heuristics, respectively. Both heuristics lead to significant optimality gaps when $\lambda^{*} t=40$. Even when $n=20$, a regret of about four percent remains for both heuristics. This shows that for a given starting inventory level $(n)$, increasing the demand potential over the season (increasing $\lambda^{*}$ or $t$ ) reduces the effectiveness of fixed-price heuristics, especially when the price is not optimized.

In general, dynamic pricing heuristics offer important improvements over FP and OFP heuristics and generate nearoptimal results. $R R$ heuristic performs better than OFP heuristic 
except five instances and its worst performance is $97.2 \%$ when $n=8$ and $\lambda^{*} t=10$. In contrast to fixed-price heuristics, $R R$ performs better when the demand potential is larger. When $\lambda^{*} t=40$, $R R$ has a near-optimal performance with minimum performance at $99.34 \%$.

$R A$ heuristic has an outstanding performance in all instances. It performs better than $F P$, OFP and $R R$ heuristics in all problems, and its worst performance is as high as $99.84 \%$ (when $n=10$ and $\lambda^{*} t=10$ ). $R A$ leads to an average of $3.97 \%$ and $7.44 \%$ improvement over FP heuristic for $\lambda^{*} t=10$ and $\lambda^{*} t=40$ cases, respectively. The improvement over OFP heuristic is, on the average, $2.22 \%$ and $5.23 \%$ for these cases. The results in Table 2 also show that combining the upper and lower bounds when approximating the revenue is important. These bounds, when used alone in approximating the optimal revenue $\left(J_{R A}^{D}\right.$ and $\left.J_{R A}^{H}\right)$, do not lead to a consistent and comparable performance.

A similar study is carried out for the linear price response function in Table 3. In particular, we used $a=2$ and $b=1$ leading to $\lambda^{*}=p^{*}=1$. The performance of $F P$ heuristic in the linear demand case is generally worse than the case of exponential demand. For $\lambda^{*} t=10$, the worst performance is at $72.06 \%$ when $n=1$. The OFP heuristic, on the other hand, performs better with the linear price response function. The worst performance is $96.66 \%$ when $n=3$. Increasing the demand potential $\lambda^{*} t$ to 40 has a more dramatic effect on FP heuristic in the case of linear price response function. For all values of $n, F P$ heuristic performs

Table 2

Performance of dynamic and fixed price heuristics, exponential, $a=e$.

\begin{tabular}{|c|c|c|c|c|c|c|c|c|c|c|c|c|c|c|}
\hline \multirow[t]{2}{*}{$n$} & \multicolumn{7}{|l|}{$\lambda^{*} t=10$} & \multicolumn{7}{|l|}{$\lambda^{*} t=40$} \\
\hline & $J^{*}$ & $J_{F P} / J^{*}$ & $J_{O F P} / J^{*}$ & $J_{R R} / J^{*}$ & $J_{R A}^{D} / J^{*}$ & $J_{R A}^{H} / J^{*}$ & $J_{R A} / J^{*}$ & $J^{*}$ & $J_{F P} / J^{*}$ & $J_{O F P} / J^{*}$ & $J_{R R} / J^{*}$ & $J_{R A}^{D} / J^{*}$ & $J_{R A}^{H} / J^{*}$ & $J_{R A} / J^{*}$ \\
\hline 1 & 2.3979 & 0.8706 & 0.9451 & 0.9866 & 0.9122 & 1.0000 & 1.0000 & 3.3327 & 0.7981 & 0.9343 & 0.9976 & 0.8974 & 1.0000 & 1.0000 \\
\hline 2 & 4.1109 & 0.9259 & 0.9468 & 0.9841 & 0.9644 & 0.9767 & 0.9998 & 6.7346 & 0.8654 & 0.9365 & 0.9973 & 0.9502 & 0.9759 & 0.9996 \\
\hline 3 & 5.4279 & 0.9452 & 0.9500 & 0.9817 & 0.9800 & 0.9698 & 0.9998 & 9.3508 & 0.8938 & 0.9387 & 0.9971 & 0.9687 & 0.9622 & 0.9993 \\
\hline 4 & 6.4682 & 0.9535 & 0.9537 & 0.9793 & 0.9862 & 0.9704 & 0.9997 & 11.6799 & 0.9101 & 0.9407 & 0.9969 & 0.9779 & 0.9536 & 0.9993 \\
\hline 5 & 7.2982 & 0.9564 & 0.9578 & 0.9769 & 0.9885 & 0.9745 & 0.9995 & 13.7866 & 0.9209 & 0.9425 & 0.9967 & 0.9834 & 0.9481 & 0.9993 \\
\hline 6 & 7.9609 & 0.9558 & 0.9621 & 0.9748 & 0.9889 & 0.9800 & 0.9993 & 15.7117 & 0.9286 & 0.9442 & 0.9965 & 0.9870 & 0.9445 & 0.9993 \\
\hline 7 & 8.4869 & 0.9523 & 0.9667 & 0.9730 & 0.9883 & 0.9855 & 0.9990 & 17.4834 & 0.9346 & 0.9458 & 0.9963 & 0.9894 & 0.9423 & 0.9994 \\
\hline 8 & 8.8998 & 0.9460 & 0.9713 & 0.9720 & 0.9872 & 0.9903 & 0.9987 & 19.1223 & 0.9393 & 0.9473 & 0.9960 & 0.9912 & 0.9412 & 0.9994 \\
\hline 9 & 9.2190 & 0.9369 & 0.9759 & 0.9724 & 0.9864 & 0.9940 & 0.9985 & 20.6443 & 0.9431 & 0.9487 & 0.9958 & 0.9925 & 0.9410 & 0.9995 \\
\hline 10 & 9.4605 & 0.9248 & 0.9805 & 0.9753 & 0.9865 & 0.9964 & 0.9984 & 22.0619 & 0.9463 & 0.9501 & 0.9956 & 0.9935 & 0.9414 & 0.9996 \\
\hline 11 & 9.6387 & 0.9509 & 0.9847 & 0.9807 & 0.9886 & 0.9978 & 0.9988 & 23.3850 & 0.9490 & 0.9514 & 0.9954 & 0.9943 & 0.9424 & 0.9996 \\
\hline 12 & 9.7662 & 0.9696 & 0.9886 & 0.9863 & 0.9916 & 0.9984 & 0.9992 & 24.6221 & 0.9513 & 0.9527 & 0.9952 & 0.9949 & 0.9438 & 0.9996 \\
\hline 13 & 9.8544 & 0.9821 & 0.9919 & 0.9911 & 0.9945 & 0.9986 & 0.9995 & 25.7803 & 0.9533 & 0.9540 & 0.9950 & 0.9954 & 0.9456 & 0.9997 \\
\hline 14 & 9.9129 & 0.9899 & 0.9946 & 0.9946 & 0.9966 & 0.9986 & 0.9997 & 26.8654 & 0.9550 & 0.9553 & 0.9948 & 0.9957 & 0.9478 & 0.9997 \\
\hline 15 & 9.9500 & 0.9946 & 0.9966 & 0.9969 & 0.9981 & 0.9985 & 0.9998 & 27.8827 & 0.9565 & 0.9565 & 0.9946 & 0.9960 & 0.9502 & 0.9997 \\
\hline 16 & 9.9726 & 0.9973 & 0.9980 & 0.9983 & 0.9990 & 0.9986 & 0.9998 & 28.8367 & 0.9578 & 0.9578 & 0.9943 & 0.9962 & 0.9528 & 0.9997 \\
\hline 17 & 9.9856 & 0.9987 & 0.9989 & 0.9992 & 0.9995 & 0.9986 & 0.9998 & 29.7314 & 0.9589 & 0.9590 & 0.9941 & 0.9963 & 0.9557 & 0.9997 \\
\hline 18 & 9.9928 & 0.9994 & 0.9995 & 0.9996 & 0.9998 & 0.9988 & 0.9998 & 30.5703 & 0.9599 & 0.9602 & 0.9939 & 0.9964 & 0.9586 & 0.9997 \\
\hline 19 & 9.9965 & 0.9997 & 0.9997 & 0.9998 & 0.9999 & 0.9989 & 0.9998 & 31.3567 & 0.9607 & 0.9615 & 0.9937 & 0.9965 & 0.9617 & 0.9997 \\
\hline 20 & 9.9984 & 0.9999 & 0.9999 & 0.9999 & 1.0000 & 0.9991 & 0.9999 & 32.0934 & 0.9614 & 0.9627 & 0.9934 & 0.9965 & 0.9649 & 0.9997 \\
\hline AVG & 8.4399 & 0.9625 & 0.9781 & 0.9861 & 0.9868 & 0.9912 & 0.9994 & 21.0516 & 0.9322 & 0.9500 & 0.9955 & 0.9845 & 0.9537 & 0.9996 \\
\hline
\end{tabular}

Table 3

Performance of dynamic and fixed price heuristics, linear, $a=2, b=1$.

\begin{tabular}{|c|c|c|c|c|c|c|c|c|c|c|c|c|c|c|}
\hline \multirow[t]{2}{*}{$n$} & \multicolumn{7}{|l|}{$\lambda^{*} t=10$} & \multicolumn{7}{|l|}{$\lambda^{*} t=40$} \\
\hline & $J^{*}$ & $J_{F P} / J^{*}$ & $J_{O F P} / J^{*}$ & $J_{R R} / J^{*}$ & $J_{R A}^{D} / J^{*}$ & $J_{R A}^{H} / J^{*}$ & $J_{R A} / J^{*}$ & $J^{*}$ & $J_{F P} / J^{*}$ & $J_{O F P} / J^{*}$ & $J_{R R} / J^{*}$ & $J_{R A}^{D} / J^{*}$ & $J_{R A}^{H} / J^{*}$ & $J_{R A} / J^{*}$ \\
\hline 1 & 1.6667 & 0.7206 & 0.9695 & 0.9798 & 0.8811 & 1.0000 & 1.0000 & 1.9048 & 0.6554 & 0.9801 & 0.9836 & 0.9039 & 1.0000 & 1.0000 \\
\hline 2 & 3.1325 & 0.8382 & 0.9674 & 0.9858 & 0.9305 & 0.9857 & 0.9995 & 3.7508 & 0.7584 & 0.9779 & 0.9861 & 0.9331 & 0.9925 & 0.9990 \\
\hline 3 & 4.4164 & 0.8961 & 0.9666 & 0.9892 & 0.9552 & 0.9768 & 0.9997 & 5.5421 & 0.8086 & 0.9762 & 0.9879 & 0.9479 & 0.9843 & 0.9984 \\
\hline 4 & 5.5307 & 0.9311 & 0.9670 & 0.9908 & 0.9700 & 0.9738 & 0.9998 & 7.2807 & 0.8399 & 0.9748 & 0.9893 & 0.9572 & 0.9765 & 0.9981 \\
\hline 5 & 6.4857 & 0.9535 & 0.9682 & 0.9909 & 0.9793 & 0.9750 & 0.9998 & 8.9678 & 0.8620 & 0.9736 & 0.9905 & 0.9638 & 0.9696 & 0.9981 \\
\hline 6 & 7.2917 & 0.9670 & 0.9702 & 0.9899 & 0.9850 & 0.9788 & 0.9997 & 10.6040 & 0.8786 & 0.9726 & 0.9915 & 0.9688 & 0.9634 & 0.9982 \\
\hline 7 & 7.9597 & 0.9729 & 0.9729 & 0.9879 & 0.9880 & 0.9836 & 0.9995 & 12.1901 & 0.8918 & 0.9717 & 0.9923 & 0.9728 & 0.9581 & 0.9983 \\
\hline 8 & 8.5017 & 0.9716 & 0.9762 & 0.9855 & 0.9890 & 0.9885 & 0.9991 & 13.7265 & 0.9026 & 0.9710 & 0.9930 & 0.9760 & 0.9536 & 0.9986 \\
\hline 9 & 8.9306 & 0.9625 & 0.9797 & 0.9835 & 0.9888 & 0.9925 & 0.9986 & 15.2137 & 0.9117 & 0.9704 & 0.9937 & 0.9787 & 0.9500 & 0.9988 \\
\hline 10 & 9.2604 & 0.9448 & 0.9834 & 0.9838 & 0.9887 & 0.9955 & 0.9982 & 16.6519 & 0.9195 & 0.9699 & 0.9942 & 0.9810 & 0.9470 & 0.9990 \\
\hline 11 & 9.5059 & 0.9642 & 0.9871 & 0.9869 & 0.9903 & 0.9974 & 0.9984 & 18.0415 & 0.9262 & 0.9695 & 0.9947 & 0.9830 & 0.9447 & 0.9992 \\
\hline 12 & 9.6821 & 0.9780 & 0.9905 & 0.9907 & 0.9929 & 0.9984 & 0.9990 & 19.3829 & 0.9321 & 0.9692 & 0.9951 & 0.9847 & 0.9431 & 0.9994 \\
\hline 13 & 9.8035 & 0.9871 & 0.9934 & 0.9939 & 0.9953 & 0.9989 & 0.9994 & 20.6763 & 0.9374 & 0.9689 & 0.9955 & 0.9863 & 0.9421 & 0.9995 \\
\hline 14 & 9.8836 & 0.9929 & 0.9957 & 0.9964 & 0.9972 & 0.9990 & 0.9997 & 21.9221 & 0.9420 & 0.9688 & 0.9958 & 0.9876 & 0.9417 & 0.9996 \\
\hline 15 & 9.9340 & 0.9962 & 0.9974 & 0.9979 & 0.9984 & 0.9991 & 0.9998 & 23.1205 & 0.9463 & 0.9687 & 0.9961 & 0.9888 & 0.9418 & 0.9997 \\
\hline 16 & 9.9642 & 0.9981 & 0.9985 & 0.9989 & 0.9992 & 0.9991 & 0.9998 & 24.2718 & 0.9501 & 0.9687 & 0.9964 & 0.9899 & 0.9424 & 0.9998 \\
\hline 17 & 9.9814 & 0.9991 & 0.9992 & 0.9995 & 0.9996 & 0.9991 & 0.9999 & 25.3764 & 0.9535 & 0.9688 & 0.9965 & 0.9909 & 0.9434 & 0.9998 \\
\hline 18 & 9.9908 & 0.9996 & 0.9996 & 0.9997 & 0.9998 & 0.9992 & 0.9999 & 26.4346 & 0.9567 & 0.9690 & 0.9967 & 0.9917 & 0.9449 & 0.9998 \\
\hline 19 & 9.9956 & 0.9998 & 0.9998 & 0.9999 & 0.9999 & 0.9993 & 0.9999 & 27.4466 & 0.9595 & 0.9692 & 0.9968 & 0.9925 & 0.9468 & 0.9998 \\
\hline 20 & 9.9980 & 0.9999 & 0.9999 & 0.9999 & 1.0000 & 0.9994 & 0.9999 & 28.4130 & 0.9621 & 0.9695 & 0.9969 & 0.9932 & 0.9490 & 0.9998 \\
\hline AVG & 8.0958 & 0.9537 & 0.9841 & 0.9915 & 0.9814 & 0.9920 & 0.9995 & 16.5459 & 0.8947 & 0.9714 & 0.9931 & 0.9736 & 0.9567 & 0.9991 \\
\hline
\end{tabular}


worse with larger demand potential. For $n=1$, the performance goes down to $65.54 \%$. When $\lambda^{*} t$ is increased from 10 to 40 , the average reduction in performance is about $6.28 \%$. The OFP heuristic, on the other hand, performs better with $\lambda^{*} t=40$ for smaller values of $n$, and performs worse for larger values of $n$. The average reduction in performance is $1.27 \%$. A regret in the range of 3-4\% still remains even for large values of $n$ for both heuristics.

Again, in general, dynamic pricing heuristics offer important improvements over FP and OFP heuristics and perform close to optimal. RR heuristic performs better than OFP heuristic except one instance and its worst performance is $97.98 \%$ when $n=1$ and $\lambda^{*} t=10$. When $\lambda^{*} t=40, R R$ has a near-optimal performance with minimum performance at $98.36 \%$.

$R A$ heuristic has an outstanding performance for the linear demand case. It performs better than $F P, O F P$ and $R R$ heuristics for all instances. Its minimum performance is $99.81 \%$ when $n=5$ and $\lambda^{*} t=40$. RA leads to an average of $5.42 \%$ and $12.65 \%$ improvement over FP heuristic for $\lambda^{*} t=10$ and $\lambda^{*} t=40$ cases, respectively. The improvement over OFP heuristic is, on the average, $1.58 \%$, and $2.85 \%$ for these cases.

Finally, in Table 4, we report the results for the logit price response function. We use $b=W(1 / e)+1$ and $a=1+e^{-W(1 / e)-1} / e^{-W(1 / e)-1}$, again leading to $p^{*}=1$ and $\lambda^{*}=1$.

The performances of FP and OFP heuristics are usually similar to what is observed for the exponential price response function. The worst performances of $F P$ and OFP heuristics for $\lambda^{*} t=10$ are $85.06 \%$ and $94.52 \%$, respectively, when $n=1$. Increasing the demand potential has a negative effect on the performance for both heuristics. Worst performances go down to $78.27 \%$ and 93.50\% for FP and OFP heuristics, respectively. On the average, increasing the demand potential $\lambda^{*} t$ from 10 to 40 reduces the performance by $3.63 \%$ and $2.80 \%$ for $F P$ and OFP, respectively.

Once again, dynamic pricing heuristics offer significant improvements over fixed-price heuristics. $R R$ heuristic performs better than OFP heuristic in all instances except for three. When $\lambda^{*} t=10$, the worst performance of $R R$ heuristic is $97.64 \%$. When $\lambda^{*} t=40$, the performance is very close to optimal with minimum at $99.53 \%$

$R A$ heuristic has a remarkable performance with the logit price response function. Once again, it performs better than $F P$, OFP and
$R R$ heuristics in all instances. The minimum performance is 99.83\% when $\lambda^{*} t=10$ and $n=10$. $R A$ heuristic offers an average performance improvement of $3.97 \%$ and $7.99 \%$ over FP heuristic for $\lambda^{*} t=10$ and $\lambda^{*} t=40$ cases, respectively. The improvement over OFP heuristic is, on the average, $2.20 \%$, and $5.15 \%$ for these cases.

In order to better understand the impact of demand potential on performance of heuristic pricing policies, we provide Fig. 5, which shows the performance of $F P, O F P, R A$ and $R R$ heuristics as a function of $t$ for the three demand functions with $n=5$ and $\lambda^{*}=p^{*}=1$.

For all demand functions, when $t$ is very small, the performance of all heuristics are close to optimal. This is expected since all four heuristics tend to use an intensity that minimizes the instantaneous revenue rate and this is optimal. The performance of FP heuristic first goes down and after $t=n / \lambda^{*}=5$ (when the intensity switches from $\lambda^{*}$ to $\lambda^{0}$ ) goes back up again. However, after a threshold, the performance of $F P$ is a decreasing in $t$. The performance of OFP heuristic tends to deteriorate as $t$ increases for an extended range of $t$ values. When $t$ is considerably large, the performance is rather flat and then increases as $t$ increases. $R R$ heuristic performs better than $F P$, but the impact of $t$ is similar for the initial part. The performance dips at $t=n / \lambda^{*}=5$. However, unlike $F P$, performance of $R R$ is monotone increasing in $t$ after this point. $R A$ heuristic has a consistently very strong performance for all demand functions and all values of $t$ again with minimum at 99.8\%. It performs better than all heuristics for all demand functions and all values of $t$.

\subsubsection{Larger problems}

The numerical analysis so far shows that FP and OFP heuristics have important regrets, especially for small and moderate values of starting inventory. In contrast, dynamic pricing heuristics and especially $R A$ heuristic, perform very close to optimal dynamic pricing policy. A critical question is whether these results are valid when $n$ is larger, as in certain problems experienced in practice. In order to answer this question, we use a continuous price version of an example used in GvR (Section 4). Consider a flight with $n=300$ seats on sale $t=360$ days prior to departure. If

Table 4

Performance of dynamic and fixed price heuristics, logit, $b=W(1 / e)+1, a=1+e^{-W(1 / e)-1} / e^{-W(1 / e)-1}$.

\begin{tabular}{|c|c|c|c|c|c|c|c|c|c|c|c|c|c|c|}
\hline \multirow[t]{2}{*}{$n$} & \multicolumn{7}{|l|}{$\lambda^{*} t=10$} & \multicolumn{7}{|l|}{$\lambda^{*} t=40$} \\
\hline & $J^{*}$ & $J_{F P} / J^{*}$ & $J_{O F P} / J^{*}$ & $J_{R R} / J^{*}$ & $J_{R A}^{D} / J^{*}$ & $J_{R A}^{H} / J^{*}$ & $J_{R A} / J^{*}$ & $J^{*}$ & $J_{F P} / J^{*}$ & $J_{O F P} / J^{*}$ & $J_{R R} / J^{*}$ & $J_{R A}^{D} / J^{*}$ & $J_{R A}^{H} / J^{*}$ & $J_{R A} / J^{*}$ \\
\hline 1 & 2.2116 & 0.8506 & 0.9452 & 0.9912 & 0.9046 & 1.0000 & 1.0000 & 3.2896 & 0.7827 & 0.9350 & 0.9985 & 0.8951 & 1.0000 & 1.0000 \\
\hline 2 & 3.8558 & 0.9141 & 0.9475 & 0.9894 & 0.9592 & 0.9771 & 0.9998 & 6.0291 & 0.8533 & 0.9377 & 0.9983 & 0.9473 & 0.9770 & 0.9995 \\
\hline 3 & 5.1587 & 0.9391 & 0.9507 & 0.9874 & 0.9769 & 0.9695 & 0.9998 & 8.4433 & 0.8837 & 0.9400 & 0.9982 & 0.9660 & 0.9634 & 0.9992 \\
\hline 4 & 6.2138 & 0.9518 & 0.9543 & 0.9853 & 0.9846 & 0.9693 & 0.9997 & 10.6244 & 0.9015 & 0.9421 & 0.9980 & 0.9755 & 0.9545 & 0.9992 \\
\hline 5 & 7.0737 & 0.9582 & 0.9582 & 0.9830 & 0.9882 & 0.9730 & 0.9996 & 12.6229 & 0.9135 & 0.9439 & 0.9979 & 0.9813 & 0.9484 & 0.9992 \\
\hline 6 & 7.7727 & 0.9604 & 0.9624 & 0.9807 & 0.9894 & 0.9784 & 0.9994 & 14.4706 & 0.9223 & 0.9455 & 0.9978 & 0.9851 & 0.9442 & 0.9992 \\
\hline 7 & 8.3361 & 0.9588 & 0.9669 & 0.9785 & 0.9894 & 0.9841 & 0.9992 & 16.1896 & 0.9291 & 0.9470 & 0.9976 & 0.9877 & 0.9414 & 0.9993 \\
\hline 8 & 8.7842 & 0.9536 & 0.9715 & 0.9768 & 0.9886 & 0.9892 & 0.9988 & 17.7959 & 0.9346 & 0.9484 & 0.9975 & 0.9897 & 0.9396 & 0.9994 \\
\hline 9 & 9.1339 & 0.9444 & 0.9761 & 0.9764 & 0.9877 & 0.9932 & 0.9985 & 19.3019 & 0.9391 & 0.9498 & 0.9973 & 0.9912 & 0.9387 & 0.9994 \\
\hline 10 & 9.4006 & 0.9307 & 0.9806 & 0.9783 & 0.9876 & 0.9959 & 0.9983 & 20.7173 & 0.9429 & 0.9510 & 0.9972 & 0.9924 & 0.9385 & 0.9995 \\
\hline 11 & 9.5984 & 0.9549 & 0.9849 & 0.9829 & 0.9894 & 0.9975 & 0.9986 & 22.0500 & 0.9461 & 0.9522 & 0.9970 & 0.9934 & 0.9389 & 0.9996 \\
\hline 12 & 9.7404 & 0.9721 & 0.9888 & 0.9879 & 0.9922 & 0.9984 & 0.9991 & 23.3063 & 0.9489 & 0.9534 & 0.9969 & 0.9941 & 0.9398 & 0.9996 \\
\hline 13 & 9.8385 & 0.9836 & 0.9921 & 0.9921 & 0.9948 & 0.9987 & 0.9995 & 24.4919 & 0.9514 & 0.9546 & 0.9967 & 0.9947 & 0.9411 & 0.9997 \\
\hline 14 & 9.9036 & 0.9909 & 0.9948 & 0.9952 & 0.9969 & 0.9987 & 0.9997 & 25.6112 & 0.9536 & 0.9557 & 0.9965 & 0.9952 & 0.9428 & 0.9997 \\
\hline 15 & 9.9449 & 0.9951 & 0.9968 & 0.9973 & 0.9982 & 0.9987 & 0.9998 & 26.6684 & 0.9555 & 0.9568 & 0.9964 & 0.9956 & 0.9449 & 0.9997 \\
\hline 16 & 9.9699 & 0.9975 & 0.9981 & 0.9985 & 0.9991 & 0.9988 & 0.9998 & 27.6670 & 0.9572 & 0.9580 & 0.9962 & 0.9959 & 0.9472 & 0.9998 \\
\hline 17 & 9.9843 & 0.9988 & 0.9990 & 0.9993 & 0.9995 & 0.9988 & 0.9998 & 28.6100 & 0.9588 & 0.9591 & 0.9960 & 0.9962 & 0.9498 & 0.9998 \\
\hline 18 & 9.9921 & 0.9994 & 0.9995 & 0.9996 & 0.9998 & 0.9989 & 0.9998 & 29.5003 & 0.9601 & 0.9602 & 0.9958 & 0.9964 & 0.9526 & 0.9998 \\
\hline 19 & 9.9962 & 0.9998 & 0.9998 & 0.9998 & 0.9999 & 0.9991 & 0.9998 & 30.3402 & 0.9613 & 0.9613 & 0.9956 & 0.9965 & 0.9555 & 0.9998 \\
\hline 20 & 9.9983 & 0.9999 & 0.9999 & 0.9999 & 1.0000 & 0.9992 & 0.9999 & 31.1320 & 0.9624 & 0.9625 & 0.9953 & 0.9966 & 0.9586 & 0.9998 \\
\hline AVG & 8.3454 & 0.9627 & 0.9784 & 0.9890 & 0.9863 & 0.9908 & 0.9994 & 19.9431 & 0.9279 & 0.9507 & 0.9970 & 0.9833 & 0.9508 & 0.9996 \\
\hline
\end{tabular}




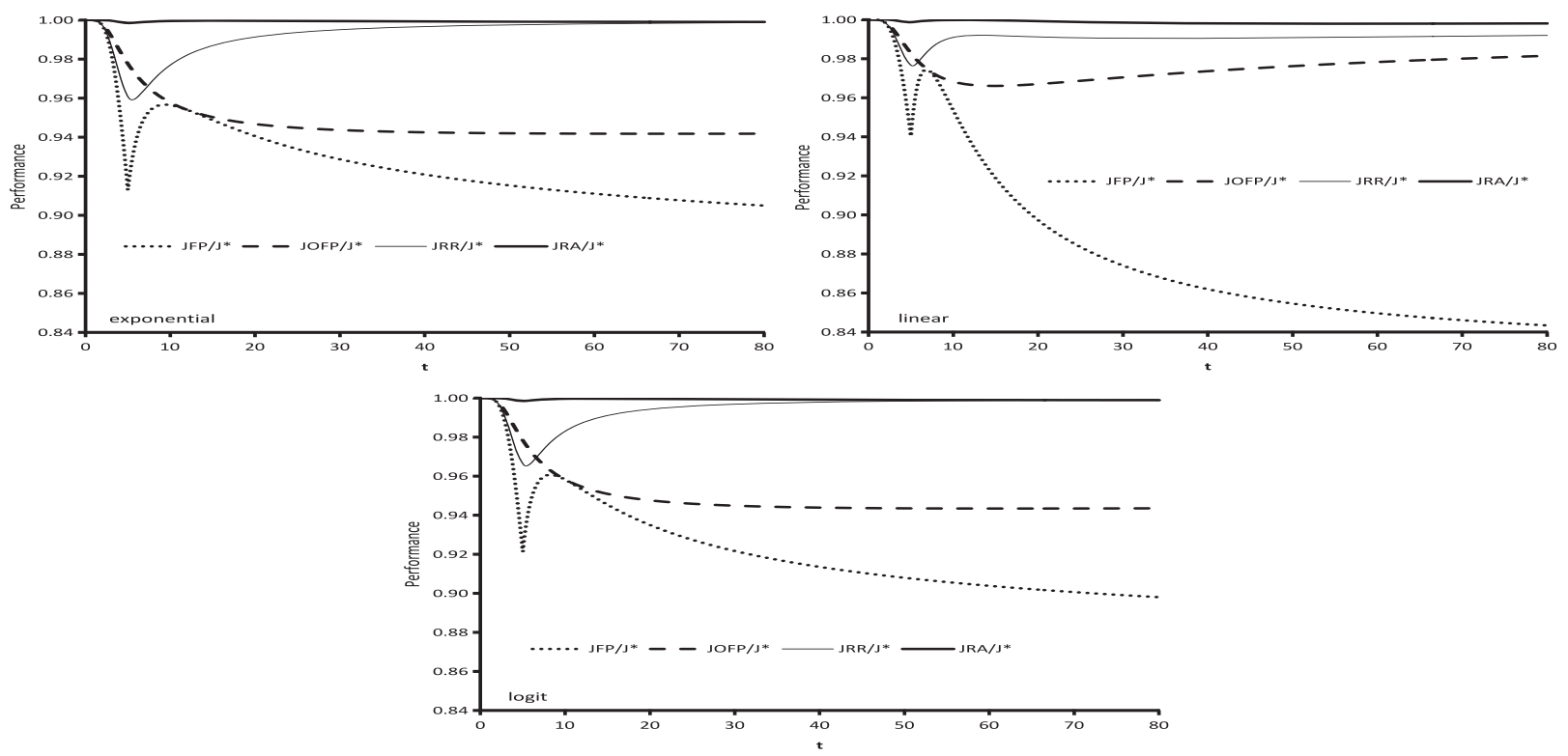

Fig. 5. Performance of fixed price heuristics, $n=5, p^{*}=\lambda^{*}=1$.

Table 5

Performance of pricing heuristics for large $n$ and $t$.

\begin{tabular}{|c|c|c|c|c|c|c|c|c|c|c|c|}
\hline Demand function & $n$ & $t$ & $J^{*}$ & $J_{F P}$ & $J_{F P} / J^{*}$ & $J_{O F P}$ & $J_{O F P} / J^{*}$ & $J_{R R}$ & $J_{R R} / J^{*}$ & $J_{R A}$ & $J_{R A} / J^{*}$ \\
\hline \multirow[t]{4}{*}{ Exponential } & 300 & 360 & $\$ 71,766$ & $\$ 70,367$ & 0.9805 & $\$ 71,435$ & 0.9954 & $\$ 71,633$ & 0.9981 & $\$ 71,749$ & 0.9998 \\
\hline & 300 & 720 & $\$ 119,306$ & $\$ 117,262$ & 0.9829 & $\$ 117,306$ & 0.9832 & $\$ 119,254$ & 0.9996 & $\$ 119,303$ & 1.0000 \\
\hline & 150 & 180 & $\$ 35,785$ & $\$ 34,840$ & 0.9736 & $\$ 35,546$ & 0.9933 & $\$ 35,668$ & 0.9967 & $\$ 35,770$ & 0.9996 \\
\hline & 150 & 360 & $\$ 59,372$ & $\$ 58,059$ & 0.9779 & $\$ 58,088$ & 0.9784 & $\$ 59,321$ & 0.9991 & $\$ 59,369$ & 0.9999 \\
\hline \multirow[t]{4}{*}{ Linear } & 300 & 360 & $\$ 75,307$ & $\$ 74,592$ & 0.9905 & $\$ 75,101$ & 0.9973 & $\$ 75,213$ & 0.9988 & $\$ 75,280$ & 0.9996 \\
\hline & 300 & 720 & $\$ 114,643$ & $\$ 112,743$ & 0.9834 & $\$ 112,936$ & 0.9851 & $\$ 114,611$ & 0.9997 & $\$ 114,639$ & 1.0000 \\
\hline & 150 & 180 & $\$ 37,549$ & $\$ 36,961$ & 0.9843 & $\$ 37,376$ & 0.9954 & $\$ 37,459$ & 0.9976 & $\$ 37,525$ & 0.9994 \\
\hline & 150 & 360 & $\$ 57,033$ & $\$ 55,822$ & 0.9788 & $\$ 55,956$ & 0.9811 & $\$ 57,002$ & 0.9995 & $\$ 57,030$ & 0.9999 \\
\hline \multirow[t]{4}{*}{ Logit } & 300 & 360 & $\$ 72,582$ & $\$ 71,146$ & 0.9802 & $\$ 72,289$ & 0.9960 & $\$ 71,897$ & 0.9906 & $\$ 72,557$ & 0.9997 \\
\hline & 300 & 720 & $\$ 118,293$ & $\$ 116,285$ & 0.9830 & $\$ 116,290$ & 0.9831 & $\$ 118,192$ & 0.9991 & $\$ 118,290$ & 1.0000 \\
\hline & 150 & 180 & $\$ 36,191$ & $\$ 35,226$ & 0.9733 & $\$ 35,971$ & 0.9939 & $\$ 35,590$ & 0.9834 & $\$ 36,171$ & 0.9995 \\
\hline & 150 & 360 & $\$ 58,861$ & $\$ 57,575$ & 0.9782 & $\$ 57,579$ & 0.9782 & $\$ 58,760$ & 0.9983 & $\$ 58,859$ & 1.0000 \\
\hline
\end{tabular}

the price is $p_{1}=\$ 198$, the demand rate is $\lambda_{1}=1$ passenger per day. If the price is $p_{2}=\$ 358$, the demand rate is $\lambda_{2}=0.5$ passenger per day. These data points correspond to parameters $a=2.35790$ (and a scaling factor $\alpha=0.004332$ ), $(a, b)=(518 / 320,1 / 320)$, and $(a, b)=(3.87534,0.00533)$ for the exponential, linear and logit demand functions, respectively. The expected revenues for the optimal policy and FP, OFP, RR and $R A$ heuristic are provided in Table 5. For each demand function, we also provide results for three other problems in which, (i) the period length is twice, (ii) the initial inventory and the period length are half, (iii) the initial inventory is half, of those of the original problem.

As expected, the performances of FP and OFP heuristics are better since the expected sales is larger than the problems considered in Tables 2-4. However, the performance of FP heuristic varies around 97-98\%. When the expected sales is not large compared to starting inventory, i.e., when $(n, t)=(300,360)$ or $(n, t)=(150,180)$, using OFP heuristic instead leads to significant improvements and near-optimal performance. However, when the expected sales is larger, i.e., when $(n, t)=(300,720)$ or $(n, t)=(150,360)$, OFP heuristic provides only slight improvements over $F P$ heuristic and its performance remains around $98 \%$. $R R$ and $R A$ heuristics offer important improvements over fixed-price heuristics for these problem instances. $R R$ performs better than OFP heuristics in all but two instances. $R A$ heuristic, on the other hand, has a truly outstanding performance. It performs better than other heuristics in all instances and very close to optimal, with a maximum regret of $0.06 \%$. We believe that the additional revenue gains in the range of $2-3 \%$ over FP and OFP heuristics through dynamic pricing are important in practice.

\subsection{Frequency of price changes}

The numerical results so far show that the dynamic pricing heuristics, particularly $R A$ heuristic, dominate the performance of fixed-price heuristics and the revenue gains through these heuristics can be very important in practice. A practical consideration is the impact of frequency of price changes. In many applications, one may find it impossible or impractical to alter the prices continuously over time and choose to use a version of these heuristics in which the prices are changed in a periodic manner. In these cases, the season is divided into a pre-specified number of periods and prices can be updated only at the beginning of these periods. For $R R$ heuristic, the deterministic problem can be resolved and the prices (or the intensities) are changed only at the beginning of each period, and this frequency of price changes corresponds to the resolving frequency. For $R A$ heuristic, the prices (or the intensities) can be determined periodically using Eq. (2) (We should note that one can attempt to solve the periodic problem optimally using a dynamic program. However, the 
Table 6

Frequency of price changes: exponential demand.

\begin{tabular}{|c|c|c|c|c|c|c|c|c|c|c|c|c|}
\hline \multirow[t]{2}{*}{$\lambda^{*} t$} & \multirow[t]{2}{*}{$n$} & \multirow[t]{2}{*}{$J_{F P}$} & \multicolumn{9}{|c|}{ Dynamic revenue rate: number of periods used } & \multirow[t]{2}{*}{$J_{R R}$} \\
\hline & & & 2 & 3 & 4 & 5 & 6 & 7 & 8 & 9 & 10 & \\
\hline \multirow[t]{6}{*}{10} & 1 & 0.8706 & 0.9591 & 0.9835 & 0.9921 & 0.9953 & 0.9961 & 0.9959 & 0.9953 & 0.9944 & 0.9934 & 0.9866 \\
\hline & 2 & 0.9259 & 0.9667 & 0.9777 & 0.9818 & 0.9834 & 0.9853 & 0.9857 & 0.9854 & 0.9848 & 0.9841 & 0.9841 \\
\hline & 3 & 0.9452 & 0.9658 & 0.9719 & 0.9756 & 0.9768 & 0.9790 & 0.9799 & 0.9800 & 0.9795 & 0.9787 & 0.9817 \\
\hline & 5 & 0.9564 & 0.9584 & 0.9639 & 0.9670 & 0.9678 & 0.9707 & 0.9718 & 0.9725 & 0.9723 & 0.9711 & 0.9769 \\
\hline & 8 & 0.9460 & 0.9508 & 0.9567 & 0.9596 & 0.9602 & 0.9637 & 0.9651 & 0.9661 & 0.9665 & 0.9646 & 0.9720 \\
\hline & 10 & 0.9248 & 0.9465 & 0.9572 & 0.9616 & 0.9629 & 0.9665 & 0.9680 & 0.9690 & 0.9695 & 0.9680 & 0.9753 \\
\hline \multirow[t]{6}{*}{40} & 1 & 0.7981 & 0.9093 & 0.9487 & 0.9675 & 0.9779 & 0.9843 & 0.9885 & 0.9913 & 0.9933 & 0.9948 & 0.9976 \\
\hline & 2 & 0.8654 & 0.9329 & 0.9574 & 0.9701 & 0.9776 & 0.9818 & 0.9849 & 0.9873 & 0.9892 & 0.9907 & 0.9973 \\
\hline & 3 & 0.8938 & 0.9426 & 0.9615 & 0.9711 & 0.9773 & 0.9806 & 0.9833 & 0.9854 & 0.9871 & 0.9886 & 0.9971 \\
\hline & 5 & 0.9209 & 0.9528 & 0.9647 & 0.9712 & 0.9759 & 0.9783 & 0.9804 & 0.9821 & 0.9835 & 0.9849 & 0.9967 \\
\hline & 8 & 0.9393 & 0.9537 & 0.9607 & 0.9649 & 0.9682 & 0.9697 & 0.9710 & 0.9722 & 0.9731 & 0.9743 & 0.9960 \\
\hline & 10 & 0.9463 & 0.9463 & 0.9497 & 0.9519 & 0.9537 & 0.9543 & 0.9550 & 0.9557 & 0.9562 & 0.9570 & 0.9956 \\
\hline AVG & & 0.9111 & 0.9487 & 0.9628 & 0.9695 & 0.9731 & 0.9759 & 0.9775 & 0.9785 & 0.9791 & 0.9792 & 0.9881 \\
\hline \multirow[t]{2}{*}{$\lambda^{*} \mathrm{t}$} & $n$ & 1 & \multicolumn{9}{|c|}{ Revenue approximation: number of periods used } & \multirow[t]{2}{*}{$J_{R A}$} \\
\hline & & & 2 & 3 & 4 & 5 & 6 & 7 & 8 & 9 & 10 & \\
\hline \multirow[t]{6}{*}{10} & 1 & 0.8461 & 0.9354 & 0.9642 & 0.9772 & 0.9841 & 0.9883 & 0.9911 & 0.9929 & 0.9943 & 0.9953 & 1.0000 \\
\hline & 2 & 0.9162 & 0.9550 & 0.9696 & 0.9774 & 0.9822 & 0.9854 & 0.9876 & 0.9893 & 0.9906 & 0.9916 & 0.9998 \\
\hline & 3 & 0.9376 & 0.9610 & 0.9717 & 0.9780 & 0.9821 & 0.9849 & 0.9870 & 0.9886 & 0.9899 & 0.9909 & 0.9998 \\
\hline & 5 & 0.9561 & 0.9688 & 0.9762 & 0.9809 & 0.9841 & 0.9863 & 0.9880 & 0.9893 & 0.9904 & 0.9913 & 0.9995 \\
\hline & 8 & 0.9698 & 0.9774 & 0.9821 & 0.9853 & 0.9875 & 0.9891 & 0.9904 & 0.9913 & 0.9920 & 0.9927 & 0.9987 \\
\hline & 10 & 0.9717 & 0.9814 & 0.9860 & 0.9886 & 0.9903 & 0.9914 & 0.9923 & 0.9930 & 0.9936 & 0.9940 & 0.9984 \\
\hline \multirow[t]{6}{*}{40} & 1 & 0.7908 & 0.9007 & 0.9402 & 0.9594 & 0.9704 & 0.9774 & 0.9821 & 0.9854 & 0.9879 & 0.9897 & 1.0000 \\
\hline & 2 & 0.8813 & 0.9342 & 0.9546 & 0.9658 & 0.9729 & 0.9778 & 0.9813 & 0.9839 & 0.9860 & 0.9876 & 0.9996 \\
\hline & 3 & 0.9096 & 0.9440 & 0.9591 & 0.9680 & 0.9739 & 0.9782 & 0.9813 & 0.9837 & 0.9856 & 0.9871 & 0.9993 \\
\hline & 5 & 0.9304 & 0.9528 & 0.9644 & 0.9715 & 0.9763 & 0.9798 & 0.9824 & 0.9845 & 0.9861 & 0.9874 & 0.9993 \\
\hline & 8 & 0.9427 & 0.9606 & 0.9698 & 0.9755 & 0.9794 & 0.9822 & 0.9843 & 0.9860 & 0.9874 & 0.9885 & 0.9994 \\
\hline & 10 & 0.9475 & 0.9644 & 0.9726 & 0.9776 & 0.9810 & 0.9835 & 0.9855 & 0.9870 & 0.9882 & 0.9893 & 0.9996 \\
\hline AVG & & 0.9167 & 0.9530 & 0.9675 & 0.9754 & 0.9804 & 0.9837 & 0.9861 & 0.9879 & 0.9893 & 0.9905 & 0.9995 \\
\hline
\end{tabular}

Table 7

Frequency of price changes: linear demand.

\begin{tabular}{|c|c|c|c|c|c|c|c|c|c|c|c|c|}
\hline \multirow[t]{2}{*}{$\lambda^{*} t$} & \multirow[t]{2}{*}{$n$} & \multirow[t]{2}{*}{$J_{F P}$} & \multicolumn{9}{|c|}{ Dynamic run-out rate: number of periods used } & \multirow[t]{2}{*}{$J_{R R}$} \\
\hline & & & 2 & 3 & 4 & 5 & 6 & 7 & 8 & 9 & 10 & \\
\hline \multirow[t]{6}{*}{10} & 1 & 0.7206 & 0.8626 & 0.9163 & 0.942 & 0.9556 & 0.9633 & 0.9675 & 0.9698 & 0.9708 & 0.971 & 0.9798 \\
\hline & 2 & 0.8382 & 0.9228 & 0.951 & 0.9638 & 0.9701 & 0.9758 & 0.9786 & 0.9798 & 0.98 & 0.9795 & 0.9858 \\
\hline & 3 & 0.8961 & 0.949 & 0.9649 & 0.9733 & 0.9769 & 0.9812 & 0.9835 & 0.9845 & 0.9844 & 0.9834 & 0.9892 \\
\hline & 5 & 0.9535 & 0.9675 & 0.9754 & 0.9795 & 0.9807 & 0.9842 & 0.9857 & 0.9868 & 0.9866 & 0.985 & 0.9909 \\
\hline & 8 & 0.9716 & 0.9689 & 0.9729 & 0.975 & 0.9751 & 0.9786 & 0.98 & 0.9809 & 0.9814 & 0.979 & 0.9855 \\
\hline & 10 & 0.9448 & 0.9576 & 0.9671 & 0.9711 & 0.9722 & 0.9757 & 0.9772 & 0.9782 & 0.9789 & 0.977 & 0.9838 \\
\hline \multirow[t]{6}{*}{40} & 1 & 0.6554 & 0.8005 & 0.862 & 0.8955 & 0.9163 & 0.9303 & 0.9402 & 0.9477 & 0.9533 & 0.9578 & 0.9836 \\
\hline & 2 & 0.7584 & 0.8587 & 0.8992 & 0.9215 & 0.9357 & 0.9427 & 0.9489 & 0.9541 & 0.9585 & 0.9622 & 0.9861 \\
\hline & 3 & 0.8086 & 0.8864 & 0.9176 & 0.9329 & 0.9437 & 0.9482 & 0.9527 & 0.9565 & 0.9602 & 0.9636 & 0.9879 \\
\hline & 5 & 0.862 & 0.916 & 0.9322 & 0.9411 & 0.9485 & 0.9505 & 0.9533 & 0.9557 & 0.9582 & 0.9612 & 0.9905 \\
\hline & 8 & 0.9026 & 0.9206 & 0.9276 & 0.9323 & 0.9368 & 0.937 & 0.9384 & 0.9396 & 0.9408 & 0.9435 & 0.993 \\
\hline & 10 & 0.9195 & 0.9079 & 0.9075 & 0.9084 & 0.9103 & 0.9096 & 0.9099 & 0.9104 & 0.9109 & 0.9127 & 0.9942 \\
\hline \multicolumn{2}{|l|}{ AVG } & 0.8526 & 0.9099 & 0.9328 & 0.9447 & 0.9518 & 0.9564 & 0.9597 & 0.9620 & 0.9637 & 0.9647 & 0.9875 \\
\hline \multirow[t]{2}{*}{$\lambda^{*} \mathrm{t}$} & $n$ & 1 & \multicolumn{9}{|c|}{ Revenue approximation: number of periods used } & \multirow[t]{2}{*}{$J_{R A}$} \\
\hline & & & 2 & 3 & 4 & 5 & 6 & 7 & 8 & 9 & 10 & \\
\hline \multirow[t]{6}{*}{10} & 1 & 0.8922 & 0.9294 & 0.9587 & 0.9799 & 0.9922 & 0.9944 & 0.9958 & 0.9968 & 0.9974 & 0.9979 & 1.0000 \\
\hline & 2 & 0.9481 & 0.9545 & 0.9669 & 0.9803 & 0.9901 & 0.9919 & 0.9932 & 0.9941 & 0.9948 & 0.9953 & 0.9995 \\
\hline & 3 & 0.9530 & 0.9612 & 0.9703 & 0.9807 & 0.9892 & 0.9910 & 0.9923 & 0.9933 & 0.9941 & 0.9947 & 0.9997 \\
\hline & 5 & 0.9601 & 0.9688 & 0.9765 & 0.9834 & 0.9891 & 0.9908 & 0.9921 & 0.9930 & 0.9937 & 0.9944 & 0.9998 \\
\hline & 8 & 0.9761 & 0.9798 & 0.9839 & 0.9877 & 0.9905 & 0.9918 & 0.9927 & 0.9934 & 0.9940 & 0.9945 & 0.9991 \\
\hline & 10 & 0.9775 & 0.9830 & 0.9870 & 0.9897 & 0.9914 & 0.9924 & 0.9932 & 0.9938 & 0.9942 & 0.9946 & 0.9982 \\
\hline \multirow[t]{6}{*}{40} & 1 & 0.8724 & 0.9150 & 0.9489 & 0.9741 & 0.9903 & 0.9930 & 0.9947 & 0.9959 & 0.9967 & 0.9973 & 1.0000 \\
\hline & 2 & 0.9509 & 0.9536 & 0.9655 & 0.9795 & 0.9907 & 0.9925 & 0.9937 & 0.9946 & 0.9952 & 0.9957 & 0.9990 \\
\hline & 3 & 0.9624 & 0.9643 & 0.9705 & 0.9804 & 0.9897 & 0.9914 & 0.9926 & 0.9934 & 0.9941 & 0.9946 & 0.9984 \\
\hline & 5 & 0.9663 & 0.9707 & 0.9750 & 0.9812 & 0.9888 & 0.9904 & 0.9916 & 0.9925 & 0.9932 & 0.9937 & 0.9981 \\
\hline & 8 & 0.9654 & 0.9715 & 0.9769 & 0.9823 & 0.9886 & 0.9902 & 0.9914 & 0.9923 & 0.9930 & 0.9936 & 0.9986 \\
\hline & 10 & 0.9645 & 0.9710 & 0.9771 & 0.9827 & 0.9887 & 0.9903 & 0.9914 & 0.9924 & 0.9931 & 0.9937 & 0.9990 \\
\hline AVG & & 0.9491 & 0.9602 & 0.9714 & 0.9818 & 0.9899 & 0.9917 & 0.9929 & 0.9938 & 0.9945 & 0.9950 & 0.9991 \\
\hline
\end{tabular}


Table 8

Frequency of price changes: logit demand.

\begin{tabular}{|c|c|c|c|c|c|c|c|c|c|c|c|c|}
\hline \multirow[t]{2}{*}{$\lambda^{*} t$} & \multirow[t]{2}{*}{$n$} & \multirow[t]{2}{*}{$J_{F P}$} & \multicolumn{9}{|c|}{ Dynamic run-out rate: number of periods used } & \multirow[t]{2}{*}{$J_{R R}$} \\
\hline & & & 2 & 3 & 4 & 5 & 6 & 7 & 8 & 9 & 10 & \\
\hline \multirow[t]{6}{*}{10} & 1 & 0.8506 & 0.9484 & 0.9778 & 0.9893 & 0.9943 & 0.9963 & 0.9969 & 0.9968 & 0.9962 & 0.9954 & 0.9912 \\
\hline & 2 & 0.9141 & 0.9632 & 0.9775 & 0.9832 & 0.9857 & 0.9884 & 0.9893 & 0.9893 & 0.9888 & 0.9881 & 0.9894 \\
\hline & 3 & 0.9391 & 0.9663 & 0.9745 & 0.9792 & 0.9809 & 0.9836 & 0.9847 & 0.9850 & 0.9846 & 0.9838 & 0.9874 \\
\hline & 5 & 0.9582 & 0.9631 & 0.9693 & 0.9726 & 0.9735 & 0.9766 & 0.9778 & 0.9786 & 0.9784 & 0.9771 & 0.9830 \\
\hline & 8 & 0.9536 & 0.9567 & 0.9622 & 0.9649 & 0.9654 & 0.9689 & 0.9703 & 0.9713 & 0.9717 & 0.9697 & 0.9768 \\
\hline & 10 & 0.9307 & 0.9502 & 0.9606 & 0.9649 & 0.9662 & 0.9697 & 0.9712 & 0.9722 & 0.9728 & 0.9711 & 0.9783 \\
\hline \multirow[t]{6}{*}{40} & 1 & 0.7827 & 0.8982 & 0.9404 & 0.9610 & 0.9727 & 0.9801 & 0.9850 & 0.9884 & 0.9909 & 0.9928 & 0.9985 \\
\hline & 2 & 0.8533 & 0.9252 & 0.9519 & 0.9659 & 0.9743 & 0.9789 & 0.9824 & 0.9852 & 0.9875 & 0.9893 & 0.9983 \\
\hline & 3 & 0.8837 & 0.9367 & 0.9574 & 0.9679 & 0.9748 & 0.9782 & 0.9811 & 0.9835 & 0.9855 & 0.9873 & 0.9982 \\
\hline & 5 & 0.9135 & 0.9490 & 0.9615 & 0.9685 & 0.9736 & 0.9760 & 0.9781 & 0.9799 & 0.9816 & 0.9832 & 0.9979 \\
\hline & 8 & 0.9346 & 0.9497 & 0.9566 & 0.9610 & 0.9645 & 0.9657 & 0.9671 & 0.9683 & 0.9693 & 0.9707 & 0.9975 \\
\hline & 10 & 0.9429 & 0.9408 & 0.9436 & 0.9455 & 0.9473 & 0.9477 & 0.9484 & 0.9490 & 0.9495 & 0.9504 & 0.9972 \\
\hline \multicolumn{2}{|l|}{ AVG } & 0.9048 & 0.9456 & 0.9611 & 0.9687 & 0.9728 & 0.9758 & 0.9777 & 0.9790 & 0.9797 & 0.9799 & 0.9911 \\
\hline \multirow[t]{2}{*}{$\lambda^{*} \mathrm{t}$} & \multirow[t]{2}{*}{$n$} & \multirow[t]{2}{*}{1} & \multicolumn{9}{|c|}{ Revenue Approximation: Number of periods used } & \multirow[t]{2}{*}{$J_{R A}$} \\
\hline & & & 2 & 3 & 4 & 5 & 6 & 7 & 8 & 9 & 10 & \\
\hline \multirow[t]{6}{*}{10} & 1 & 0.8389 & 0.9334 & 0.9636 & 0.9771 & 0.9843 & 0.9885 & 0.9913 & 0.9932 & 0.9945 & 0.9955 & 1.0000 \\
\hline & 2 & 0.9145 & 0.9547 & 0.9698 & 0.9778 & 0.9826 & 0.9858 & 0.9881 & 0.9897 & 0.9910 & 0.9920 & 0.9998 \\
\hline & 3 & 0.9364 & 0.9609 & 0.9720 & 0.9784 & 0.9826 & 0.9855 & 0.9875 & 0.9891 & 0.9903 & 0.9913 & 0.9998 \\
\hline & 5 & 0.9554 & 0.9690 & 0.9766 & 0.9813 & 0.9846 & 0.9868 & 0.9885 & 0.9898 & 0.9909 & 0.9917 & 0.9996 \\
\hline & 8 & 0.9707 & 0.9779 & 0.9825 & 0.9856 & 0.9878 & 0.9894 & 0.9906 & 0.9915 & 0.9923 & 0.9929 & 0.9988 \\
\hline & 10 & 0.9725 & 0.9813 & 0.9859 & 0.9885 & 0.9902 & 0.9914 & 0.9923 & 0.9930 & 0.9935 & 0.9940 & 0.9983 \\
\hline \multirow[t]{6}{*}{40} & 1 & 0.7816 & 0.8958 & 0.9373 & 0.9576 & 0.9692 & 0.9766 & 0.9815 & 0.9850 & 0.9876 & 0.9896 & 1.0000 \\
\hline & 2 & 0.8778 & 0.9327 & 0.9537 & 0.9653 & 0.9726 & 0.9776 & 0.9813 & 0.9840 & 0.9861 & 0.9878 & 0.9995 \\
\hline & 3 & 0.9080 & 0.9434 & 0.9588 & 0.9679 & 0.9740 & 0.9782 & 0.9814 & 0.9838 & 0.9858 & 0.9873 & 0.9992 \\
\hline & 5 & 0.9301 & 0.9528 & 0.9644 & 0.9716 & 0.9764 & 0.9800 & 0.9826 & 0.9847 & 0.9863 & 0.9877 & 0.9992 \\
\hline & 8 & 0.9427 & 0.9606 & 0.9699 & 0.9756 & 0.9795 & 0.9824 & 0.9845 & 0.9862 & 0.9876 & 0.9887 & 0.9994 \\
\hline & 10 & 0.9475 & 0.9644 & 0.9726 & 0.9777 & 0.9812 & 0.9837 & 0.9856 & 0.9872 & 0.9884 & 0.9894 & 0.9995 \\
\hline AVG & & 0.9147 & 0.9522 & 0.9673 & 0.9754 & 0.9804 & 0.9838 & 0.9863 & 0.9881 & 0.9895 & 0.9907 & 0.9994 \\
\hline
\end{tabular}

problem becomes intractable quickly. The prices that will be used by periodic versions of $R R$ and $R A$ heuristics are easily computable and in most cases, are closed-form expressions).

Table 6 shows the impact of the number of periods used on the performance of $R R$ and $R A$ heuristics for exponential demand function when $\lambda^{*}=p^{*}=1$. For the upper part of Table 6 , the third column is the performance (as a ratio of the optimal dynamic policy) of FP heuristic (no resolving). The last column is the performance of $R R$ heuristic with continuous resolving. Columns 4-12 show the performance of $R R$ heuristic when 2-10 equallength periods are used. For the lower part of Table 6 , the third column is the performance of $R A$ heuristic when the price is set at the beginning and never changed. The last column is the performance of $R A$ heuristic when the prices are continuously adjusted. Columns 4-12 show the performance of $R A$ heuristic when 2-10 equal-length periods are used.

The results in Table 6 are important. First, while resolving periodically generates better performance than FP heuristic, the impact of resolving is not monotone, i.e., resolving more often does not necessarily lead to better performance. This is especially true when starting inventory $(n)$ and demand potential $\left(\lambda^{*} t\right)$ are both small. For moderate $n$ and large $\lambda^{*} t$, resolving may provide important gains over FP heuristic (consider, for example, $n=10$ and $\lambda^{*} t=40$ ). However, in order to realize these gains, resolving has to take place very frequently; infrequent resolving generates only modest improvement. One important observation is that for $n=1$ and $\lambda^{*} t=10$, the performance of continuous resolving is worse than resolving 4-10 times throughout the horizon. Similarly for $n=2$ and $\lambda^{*} t=10$, the performance of continuous resolving is worse than resolving 5-10 times throughout the horizon.
Table 6 shows that $R A$ heuristic behaves better with respect to the frequency of price changes. Updating prices more often always leads to better performance for $R A$ heuristic. One can quickly get close to the full revenue potential of $R A$ heuristic by introducing a limited number of opportunities to update prices, especially when the expected demand is small $\left(\lambda^{*} t=10\right)$.

Table 7 extends the analysis to the linear demand function. In this case, we have negative results similar to one provided in [9] for $R R$ heuristic. For example, when $n=10$ and $\lambda^{*} t=40$, resolving, if not frequent enough, leads to a performance worse than that of $F P$ heuristic. Note that for this instance, continuous resolving provides more than eight percent improvement over FP heuristic. One can also observe the non-monotonicity of the $R R$ heuristic's performance with respect to resolving frequency in Table 7 . With linear demand function, $R A$ heuristic continues to behave nicely with respect to the frequency of price changes. Increasing frequency always leads to better performance. With linear demand, for all problems, a performance around 99\% can be obtained by using five opportunities to change the price.

Table 8 shows the results for the logit price response function. Again, $R R$ heuristic's performance is not monotone in resolving frequency. Resolving may lead to a performance worse than $F P$ heuristic, and resolving continuously may lead to a performance worse than resolving periodically. On the other hand, the performance $R A$ heuristic is monotone in the frequency of price changes also for the logit function.

We conclude that in practical settings where continuously changing prices is not possible, one should carefully fine-tune the resolving frequency for $R R$ heuristic for each problem setting as there does not seem to be any universal relationship between the resolving frequency and solution quality. The performance of $R A$ 
heuristic, on the other hand, is monotone in the frequency of price changes. One can obtain the desired performance by setting the frequency sufficiently high.

\section{Conclusion}

In this paper, we investigate the use of fixed and dynamic pricing policies for selling a fixed amount of inventory over a finite horizon. We propose two simple and computationally feasible dynamic pricing heuristics that can be used to update prices as uncertainty is resolved throughout the horizon. The first heuristic, the revenue approximation heuristic, is based on approximating the value function that arise in the dynamic programming formulation to determine optimal prices. The second heuristic, the dynamic run-out rate heuristic, is based on continuously resolving the deterministic version of the problem. Through a detailed numerical study, we demonstrate that fixedprice heuristics lead to serious shortcomings in revenue with general demand functions for moderate and small values of starting inventory when the demand potential is large. We show that these are precisely the settings in which the dynamic pricing heuristics that we propose can be effectively used to obtain nearoptimal performance. In particular, the revenue approximation heuristic has a consistently remarkable performance, leading to a maximum $0.2 \%$ optimality gap in all problems we consider. We also study the impact of changing prices periodically rather than continuously using these heuristics. We show that the revenue approximation heuristic's performance is monotone in the number of periods used and one can quickly get close to the full revenue potential of continuous price changes. Our main conclusion is that dynamic pricing heuristics lead to near-optimal performance and can provide important gains over fixed-price heuristics even when there is only normal statistical variation in demand and that their use should be given more consideration in theory and practice.

\section{References}

[1] Gallego G, van Ryzin G. Optimal dynamic pricing of inventories with stochastic demand over finite horizons. Management Science 1994;40(8): 999-1020.

[2] Kim S, Bell PC. Optimal pricing and production decisions in the presence of symmetrical and asymmetrical substitution. Omega 2011;39(5):528-538.

[3] Zhao L, Tian P, Li X. Dynamic pricing in the presence of consumer inertia. Omega 2012;40(2):137-148.

[4] Tsai WH, Hung SJ. Dynamic pricing and revenue management process in Internet retailing under uncertainty: an integrated real options approach Omega 2009;37(2):471-481.

[5] Bitran G, Caldentey R. An overview of pricing models for revenue management. Manufacturing and Service Operations Management 2003;5(3): 203-229.

[6] Elmaghraby W, Keskinocak P. Dynamic pricing in the presence of inventory considerations: Research overview, current practices and future directionsManagement Science 2003;49(10):1287-1309.

[7] Talluri K, van Ryzin G. The theory and practice of revenue management. Kluwer Academic Publishers; 2004.

[8] Gallego G, van Ryzin G. A multiproduct dynamic pricing problem and its applications to network yield management. Operations Research 1997;45(1): 24-41.

[9] Cooper WL. Aysmptotic behavior of an allocation policy for revenue management. Operations Research 2002;50(4):720-727.

[10] Secomandi N. An analysis of the control-algorithm re-solving issue in inventory and revenue management. Manufacturing and Service Operations Management 2008;10(3):468-483.

[11] Maglaras C, Meissner J. Dynamic pricing strategies for multiproduct revenue management problems. Manufacturing and Service Operations Management 2006;8(2):136-148.

[12] Zhao W, Zheng Y-S. Optimal dynamic pricing of perishable assets with nonhomogeneous demand. Management Science 2000;46(3):375-388.

[13] Phillips R. Pricing and revenue optimization. Stanford University Press; 2005

[14] Talluri K, van Ryzin G. An analysis of bid-price controls for network revenue management. Management Science 1998;44(11):1577-1593.

[15] Adelman D. Dynamic bid prices controls in revenue management. Operations Research 2007;55(4):647-661.

[16] Zhang D, Cooper WL. Pricing substitutable flights in airline revenue management. European Journal of Operational Research 2009;197:848-861. 